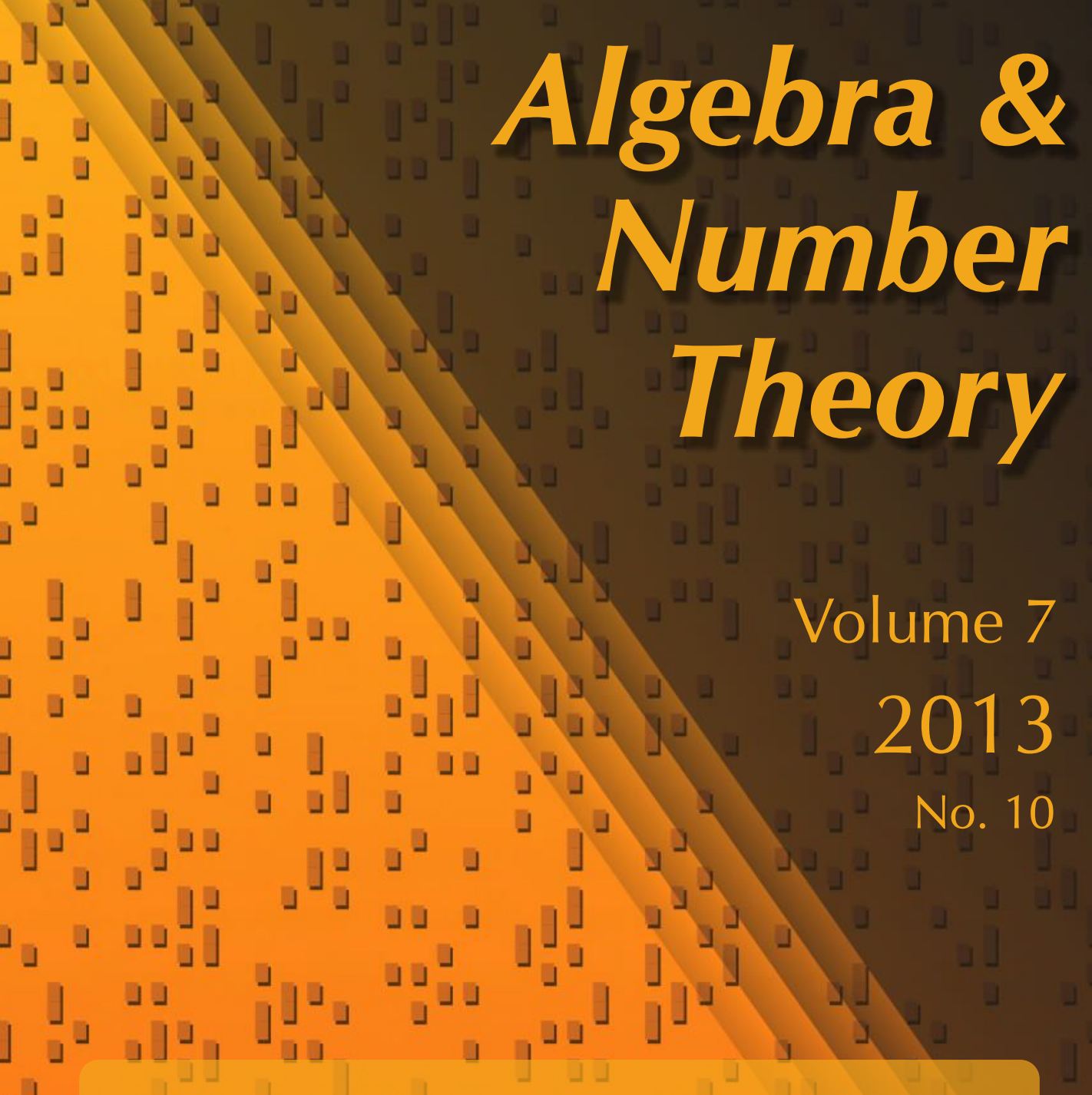

Genericity and contragredience in the local Langlands correspondence

Tasho Kaletha

J.

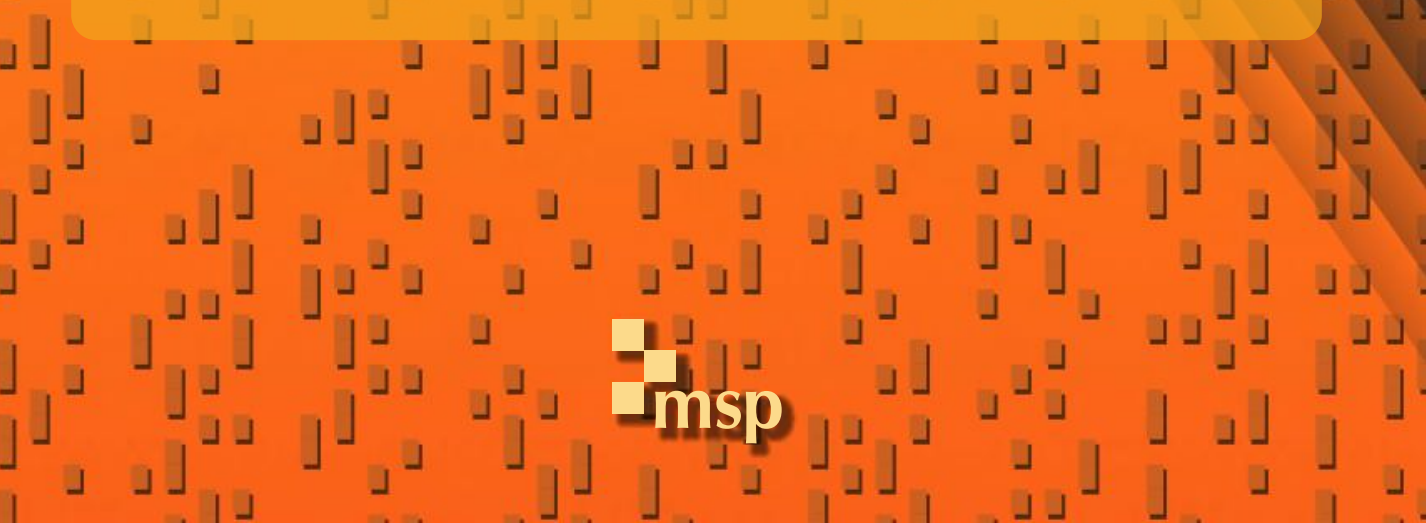




\title{
Genericity and contragredience in the local Langlands correspondence
}

\author{
Tasho Kaletha
}

\begin{abstract}
Adams, Vogan, and D. Prasad have given conjectural formulas for the behavior of the local Langlands correspondence with respect to taking the contragredient of a representation. We prove these conjectures for tempered representations of quasisplit real $K$-groups and quasisplit $p$-adic classical groups (in the sense of Arthur). We also prove a formula for the behavior of the local Langlands correspondence for these groups with respect to changes of the Whittaker data.
\end{abstract}

\section{Introduction}

The local Langlands correspondence is a conjectural relationship between certain representations of the Weil or Weil-Deligne group of a local field $F$ and finite sets, or packets, of representations of a locally compact group arising as the $F$-points of a connected reductive algebraic group defined over $F$. In characteristic zero, this correspondence is known for $F=\mathbb{R}$ and $F=\mathbb{C}$ by the work of Langlands [1989] and was later generalized and reinterpreted geometrically by Adams, Barbasch, and Vogan [Adams et al. 1992]. Furthermore, many cases are known when $F$ is a finite extension of the field $\mathbb{Q}_{p}$ of $p$-adic numbers. Most notably, the correspondence over $p$-adic fields is known when the reductive group is $\mathrm{GL}_{n}$ by work of Harris and Taylor [2001] and Henniart [2000], and has very recently been obtained for quasisplit symplectic and orthogonal groups by Arthur [2013]. Other cases include the group $U_{3}$ by work of Rogawski, $\mathrm{Sp}_{4}$ and $\mathrm{GSp}_{4}$ by work of Gan-Takeda. For general connected reductive groups, there are constructions of the correspondence for specific classes of parameters, including the classical case of unramified representations, the case of representations with Iwahori-fixed vector by work of Kazhdan-Lusztig, unipotent representations by work of Lusztig, and more recently regular depthzero supercuspidal representations by [DeBacker and Reeder 2009], very cuspidal representations [Reeder 2008], and epipelagic representations [Kaletha 2012].

MSC2010: primary 11S37; secondary 22E50.

Keywords: local Langlands correspondence, contragredient, generic, Whittaker data, $L$-packet, classical group. 
The purpose of this paper is to explore how the tempered local Langlands correspondence behaves with respects to two basic operations on the group. The first operation is that of taking the contragredient of a representation. In a recent paper, Adams and Vogan [2012] studied this question for the general (not just tempered) local Langlands correspondence for real groups. They provide a conjecture on the level of $L$-packets for any connected reductive group over a local field $F$ and prove this conjecture when $F$ is the field of real numbers. One of our main results is the fact that this conjecture holds for the tempered $L$-packets of symplectic and special orthogonal $p$-adic groups constructed by Arthur. In fact, inspired by the work of Adams and Vogan, we provide a refinement of their conjecture to the level of representations, rather than packets, for the tempered local Langlands correspondence. We prove this refinement when $G$ is either a quasisplit connected real reductive group (more generally, quasisplit real $K$-group), a quasisplit symplectic or special orthogonal $p$-adic group, and in the context of the constructions of [DeBacker and Reeder 2009] and [Kaletha 2012]. In the real case, the results of Adams and Vogan are a central ingredient in our argument. To obtain our results, we exploit the internal structure of real $L$-packets using recent results of Shelstad [2008]. In the case of quasisplit $p$-adic symplectic and special orthogonal groups, we prove a result similar to that of Adams and Vogan using Arthur's characterization of the stable characters of $L$-packets on quasisplit $p$-adic classical groups as twisted transfers of characters of $\mathrm{GL}_{n}$. After that, the argument is the same as for the real case. The constructions of [DeBacker and Reeder 2009] and [Kaletha 2012] are inspected directly.

The second basic operation that we explore is that of changing the Whittaker datum. To explain it, we need some notation. Let $F$ be a local field and $G$ a connected reductive group defined over $F$. Let $W^{\prime}$ be the Weil group of $F$ if $F=\mathbb{R}$ or the Weil-Deligne group of $F$ if $F$ is an extension of $\mathbb{Q}_{p}$. Then, if $G$ is quasisplit, it is expected that there is a bijective correspondence $(\varphi, \rho) \mapsto \pi$. The target of this correspondence is the set of equivalence classes of irreducible admissible tempered representations. The source of this correspondence is the set of pairs $(\varphi, \rho)$ where $\varphi: W^{\prime} \rightarrow{ }^{L} G$ is a tempered Langlands parameter, and $\rho$ is an irreducible representation of the finite group $\pi_{0}\left(\operatorname{Cent}(\varphi, \hat{G}) / Z(\hat{G})^{\Gamma}\right)$. Here $\hat{G}$ is the complex (connected) Langlands dual group of $G$, and ${ }^{L} G$ is the $L$-group of $G$. However, it is known that such a correspondence can in general not be unique. In order to hope for a unique correspondence, following Shahidi [1990, Section 9] one must choose a Whittaker datum for $G$, which is a $G(F)$-conjugacy class of pairs $(B, \psi)$ where $B$ is a Borel subgroup of $G$ defined over $F$ and $\psi$ is a generic character of the $F$-points of the unipotent radical of $B$. Then it is expected that there exists a bijection $(\varphi, \rho) \mapsto \pi$ as above which has the property that $\pi$ has a $(B, \psi)$-Whittaker functional precisely when $\rho=1$. Let us denote this conjectural 
correspondence by $\iota_{B, \psi}$. We are interested in how it varies when one varies the Whittaker datum $(B, \psi)$. We remark that there is a further normalization of $\iota_{B, \psi}$ that must be chosen. As described in [Kottwitz and Shelstad 2012, Section 4], it is expected that there will be two normalizations of the local Langlands correspondence for reductive groups, reflecting the two possible normalizations of the local Artin reciprocity map. Related to these normalizations are the different normalizations of the transfer factors $\Delta, \Delta^{\prime}, \Delta_{D}$ and $\Delta_{D}^{\prime}$ for ordinary and twisted endoscopy described in [Kottwitz and Shelstad 2012, Section 5].

The reason we study these two questions together is that they appear to be related. Indeed, when one studies how the pair $(\varphi, \rho)$ corresponding to a representation $\pi$ changes when one takes the contragredient of $\pi$, one is led to consider $\iota_{B, \psi}$ for different Whittaker data.

We will now go into more detail and describe our expectation for the behavior of the local Langlands correspondence with respect to taking contragredient and changing the Whittaker datum. We emphasize that we claim no originality for these conjectures. Our formula in the description of the contragredient borrows greatly from the paper of Adams and Vogan, as well as from a conversation with Robert Kottwitz, who suggested taking the contragredient of $\rho$. After the paper was written, we were informed by Dipendra Prasad that an equation closely related to (1) is stated as a conjecture in [Gan, Gross, and Prasad 2012, Section 9], and that moreover (2) is part of a more general framework of conjectures currently being developed by him under the name "relative local Langlands correspondence". We refer the reader to the draft [Prasad 2012].

We continue to assume that $F$ is either real or $p$-adic, and $G$ is a quasisplit connected reductive group defined over $F$. Fix a Whittaker datum $(B, \psi)$. For any Langlands parameter $\varphi: W^{\prime} \rightarrow{ }^{L} G$, let $S_{\varphi}=\operatorname{Cent}(\varphi, \hat{G})$. The basic form of the expected tempered local Langlands correspondence is a bijection $\iota_{B, \psi}$ from the set of pairs $(\varphi, \rho)$, where $\varphi$ is a tempered Langlands parameter and $\rho$ is an irreducible representation of $\pi_{0}\left(S_{\varphi} / Z(\hat{G})^{\Gamma}\right)$ to the set of equivalence classes of irreducible admissible tempered representations. A refinement of this correspondence is obtained when one allows $\rho$ to be an irreducible representation of $\pi_{0}\left(S_{\varphi}\right)$ rather than its quotient $\pi_{0}\left(S_{\varphi} / Z(\hat{G})^{\Gamma}\right)$. The right-hand side is then the set of equivalence classes of tuples $\left(G^{\prime}, \xi, u, \pi\right)$, where $\xi: G \rightarrow G^{\prime}$ is an inner twist, $u \in Z^{1}(F, G)$ is an element with the property $\xi^{-1} \sigma(\xi)=\operatorname{Int}(u(\sigma))$ for all $\sigma \in \Gamma$, and $\pi$ is an irreducible admissible tempered representation of $G^{\prime}(F)$. The triples $\left(G^{\prime}, \xi, u\right)$ are called pure inner twists of $G$, and the purpose of this refined version of the correspondence is to include connected reductive groups which are not quasisplit. The idea of using pure inner forms is due to Vogan, and one can find a formulation of this refinement of the correspondence in [Vogan 1993] or [DeBacker and Reeder 2009, Section 3]. A further refinement is obtained by allowing $\rho$ to be an irreducible 
algebraic representation of the complex algebraic group $\bar{S}_{\varphi}=S_{\varphi} /\left[S_{\varphi} \cap \hat{G}_{\mathrm{der}}\right]^{\circ}$. The right-hand side then is the set of equivalence classes of tuples $\left(G^{\prime}, \xi, b, \pi\right)$, where $\xi: G \rightarrow G^{\prime}$ is an inner twist and $b$ is a basic element of $Z^{1}(W, G(\bar{L}))$, where $L$ is the completion of the maximal unramified extension of $F$, and where $b$ gives rise to $\xi$ as in [Kottwitz 1997]. This further refinement was introduced by Kottwitz in an attempt to include all connected reductive groups into the correspondence (it is known that not every connected reductive group is a pure inner form of a quasisplit group). Indeed, when the center of $G$ is connected, all inner forms of $G$ come from basic elements of $Z^{1}(W, G(\bar{L}))$. Moreover, one can reduce the general case to that of connected center. An exposition of this formulation of the correspondence can be found in [Kaletha 2011].

We now let $\iota_{B, \psi}$ denote any version of the above conjectural correspondence, normalized so that $\iota_{B, \psi}(\varphi, \rho)$ is $(B, \psi)$-generic precisely when $\rho=1$. The set of Whittaker data for $G$ is a torsor for the abelian group $G_{\text {ad }}(F) / G(F)$. Dualizing Langlands' construction of a character on $G(F)$ for each element of $H^{1}(W, Z(\hat{G}))$, one obtains from each element of $G_{\mathrm{ad}}(F) / G(F)$ a character on the finite abelian group $\operatorname{ker}\left(H^{1}\left(W, Z\left(\hat{G}_{\mathrm{sc}}\right)\right) \rightarrow H^{1}(W, Z(\hat{G}))\right)$. This groups accepts a map from $\pi_{0}\left(S_{\varphi} / Z(\hat{G})^{\Gamma}\right)$ for every Langlands parameter $\varphi$. In this way, given a pair of Whittaker data $\mathfrak{w}$ and $\mathfrak{w}^{\prime}$, the element of $G_{\text {ad }}(F) / G(F)$ which conjugates $\mathfrak{w}$ to $\mathfrak{w}^{\prime}$ provides a character on $\pi_{0}\left(S_{\varphi} / Z(\hat{G})^{\Gamma}\right)$, hence also on $\pi_{0}\left(S_{\varphi}\right)$ and $\bar{S}_{\varphi}$. We denote this character by $\left(\mathfrak{w}, \mathfrak{w}^{\prime}\right)$. Then we expect that

$$
\iota_{\mathfrak{w}}(\varphi, \rho)=\iota_{\mathfrak{w}}\left(\varphi, \rho \otimes\left(\mathfrak{w}, \mathfrak{w}^{\prime}\right)^{\epsilon}\right),
$$

where $\epsilon=1$ if $\iota_{\mathfrak{w}}$ and $\iota_{\mathfrak{w}^{\prime}}$ are compatible with endoscopic transfer via the transfer factors $\Delta^{\prime}$ or $\Delta_{D}^{\prime}$, and $\epsilon=-1$ if $\iota_{\mathfrak{w}}$ and $\iota_{\mathfrak{w}}$ are compatible endoscopic transfer via the transfer factors $\Delta$ or $\Delta_{D}$.

To describe how we expect $\iota_{B, \psi}$ to behave with respect to taking contragredients, we follow [Adams and Vogan 2012] and consider the Chevalley involution on $\hat{G}$ : As is shown in [Adams and Vogan 2012], there exists a canonical element of $\operatorname{Out}(\hat{G})$ which contains all automorphisms of $\hat{G}$ that act as inversion on some maximal torus. This canonical element provides a canonical $\hat{G}$-conjugacy class of $L$-automorphisms of ${ }^{L} G$ as follows. Fix a $\Gamma$-invariant splitting of $\hat{G}$ and let $\hat{C} \in \operatorname{Aut}(\hat{G})$ be the unique lift of the canonical element of $\operatorname{Out}(\hat{G})$ which sends the fixed splitting of $\hat{G}$ to its opposite. Then $\hat{C}$ commutes with the action of $\Gamma$, and we put ${ }^{L} C$ to be the automorphism of $\hat{G} \rtimes W$ given by $\hat{C} \rtimes$ id. If we change the splitting of $\hat{G}$, there exists [Kottwitz 1984, Corollary 1.7] an element $g \in \hat{G}^{\Gamma}$ which conjugates it to the old splitting. This element also conjugates the two versions of $\hat{C}$, and hence also the two versions of ${ }^{L} C$. We conclude that $\hat{G}$-conjugacy class of ${ }^{L} C$ is indeed canonical. Thus, for any Langlands parameter $\varphi: W^{\prime} \rightarrow{ }^{L} G$, we have a well-defined (up to equivalence) Langlands parameter ${ }^{L} C \circ \varphi$. The automorphism $\hat{C}$ restricts to 
an isomorphism $S_{\varphi} \rightarrow S_{L_{C} \circ}$ and for each representation $\rho$ of $\bar{S}_{\varphi}$ we can consider the representation $\rho \circ \hat{C}^{-1}$ of $\bar{S}_{{ }_{C} \circ \varphi}$. When $\varphi$ is tempered, we expect

$$
\iota_{B, \psi}(\varphi, \rho)^{\vee}=\iota_{B, \psi^{-1}}\left({ }^{L} C \circ \varphi, \rho^{\vee} \circ \hat{C}^{-1}\right) .
$$

For this formula it is not important whether $\iota_{B, \psi}$ is normalized with respect to the classical or Deligne's normalization of the local Artin map, as long as $\iota_{B, \psi^{-1}}$ is normalized in the same way.

We will now briefly describe the contents of this paper. In Section 3, we recall the fundamental results of Arthur and Shelstad on the endoscopic classification of tempered representations of real and classical $p$-adic groups. In Section 4 we will describe more precisely the construction of the character $\left(\mathfrak{w}, \mathfrak{w}^{\prime}\right)$ alluded to in this introduction, and will then prove (1). Section 5 is devoted to the proof of (2) for tempered representations of quasisplit real $K$-groups and quasisplit symplectic and special orthogonal $p$-adic groups. Finally, in Section 6 we consider depth-zero and epipelagic $L$-packets on general $p$-adic groups and prove (2) for those cases as well.

The arguments in Sections 4 and 5 are quite general and we expect them to provide a proof of (1) and (2) for other $p$-adic groups besides symplectic and orthogonal, as soon as Arthur's work has been extended to them. For example, we expect that the case of unitary groups will follow directly from our arguments.

\section{Notation}

Throughout this paper, $F$ will denote either the field $\mathbb{R}$ or a finite extension of the field $\mathbb{Q}_{p}$. We write $W$ for the absolute Weil group of $F$, and $\Gamma$ for the absolute Galois group. We let $W^{\prime}$ stand for the Weil group of $F$ when $F=\mathbb{R}$ and for the Weil-Deligne group of $F$ if $F$ is an extension of $\mathbb{Q}_{p}$.

Given a connected reductive group $G$ defined over $F$, we will write $\hat{G}$ for the complex connected Langlands dual group of $G$, and ${ }^{L} G$ for the $L$-group. Given a maximal torus $S \subset G$, we write $R(S, G)$ for the set of roots of $S$ in $G, N(S, G)$ for the normalizer of $S$ in $G$, and $\Omega(S, G)$ for the Weyl group $N(S, G) / S$. We will write $Z(G)$ for the center of $G$, and $G_{\mathrm{sc}}$ and $G_{\mathrm{ad}}$ for the simply connected cover and the adjoint quotient of the derived subgroup $G_{\mathrm{der}}$ of $G$.

Given a finite group $\mathscr{Y}$, we will write $\operatorname{Irr}(\mathscr{Y})$ for the set of isomorphism classes of irreducible representations of $\mathscr{Y}$. The subset consisting of the one-dimensional representations will be called $\mathscr{S}^{D}$. Given a complex algebraic group $\mathscr{Y}$, we will write $\operatorname{Irr}(\mathscr{Y})$ for the set of isomorphism classes of irreducible algebraic representations of $\mathscr{Y}$.

We will use freely the language and basic constructions in the theory of endoscopy. We refer the reader to [Langlands and Shelstad 1987] and [Kottwitz and Shelstad 1999] for the foundations of the theory. 


\section{Results of Arthur and Shelstad}

In this section we will recall the results of Arthur and Shelstad on endoscopic transfer and its inversion, which will be an essential ingredient in our proofs. The formulation in the real case is slightly more complicated due to the fact that semisimple simply connected real groups can have nontrivial Galois cohomology, so we will describe the $p$-adic case first.

Let $F$ be a $p$-adic field. Arthur's results apply to groups $G$ which are either the symplectic group, or the split special odd orthogonal group, or the split or quasisplit special even orthogonal groups, as well as to products of such groups with copies of $\mathrm{GL}_{n}$. If $G$ is such a group, Arthur fixes a maximal compact subgroup $K$ of $G(F)$ and denotes by $\mathscr{H}(G)$ the Hecke algebra of smooth, compactly supported, right and left $K$-finite functions on $G(F)$. To describe the results of his that we'll need, let us first assume that $G$ has no even orthogonal factors, as the case of even orthogonal groups is slightly more subtle. Fix a Whittaker datum $(B, \psi)$. Let $\varphi: W^{\prime} \rightarrow{ }^{L} G$ be a tempered Langlands parameter and put $\mathscr{S}_{\varphi}=\pi_{0}\left(\operatorname{Cent}(\varphi, \hat{G}) / Z(\hat{G})^{\Gamma}\right)$. Arthur's recent results [2013, Section 2] imply that there exists an $L$-packet $\Pi_{\varphi}$ of representations of $G(F)$ and a canonical bijection

$$
\iota_{B, \psi}: \operatorname{Irr}\left(\mathscr{Y}_{\varphi}\right) \rightarrow \Pi_{\varphi}, \quad \rho \mapsto \pi_{\rho},
$$

which sends the trivial representation to a $(B, \psi)$-generic representation. This bijection can also be written as a pairing $\langle\cdot, \cdot\rangle: \mathscr{S}_{\varphi} \times \Pi_{\varphi} \rightarrow \mathbb{C}$, and this is the language adopted by Arthur. A semisimple element $s \in \operatorname{Cent}(\varphi, \hat{G})$ gives rise to an endoscopic datum $\mathfrak{e}=(H, \mathscr{H}, s, \xi)$ for $G$. We briefly recall the construction, following [Kottwitz and Shelstad 1999, Section 2]: $\hat{H}=\hat{G}_{s}^{\circ}, \mathscr{H}=\hat{H} \cdot \varphi(W)$, and $\xi$ is the inclusion map $\mathscr{H} \rightarrow{ }^{L} G$. The group $\mathscr{H}$ can be shown to be a split extension of $W$ by $\hat{H}$, and hence provides a homomorphism $\Gamma \rightarrow \operatorname{Out}(\hat{H})$. The group $H$ is the unique quasisplit group with complex dual $\hat{H}$ for which the homomorphism $\Gamma \rightarrow$ $\operatorname{Out}(H)$ given by the rational structure coincides under the canonical isomorphism $\operatorname{Out}(H) \cong \operatorname{Out}(\hat{H})$ with the homomorphism $\Gamma \rightarrow \operatorname{Out}(\hat{H})$ given by $\mathcal{H}$. In addition to the datum $(H, \mathscr{H}, s, \xi)$, Arthur chooses [2013, Section 1.2] an $L$-isomorphism $\xi_{H_{1}}: \mathscr{H} \rightarrow{ }^{L} H$. By construction, $\varphi$ factors through $\xi$ and we obtain $\varphi_{s}=\xi_{H_{1}} \circ \varphi$ which is a Langlands parameter for $H$. The group $H$ is again of the same type as $G$ - a product of symplectic, orthogonal, and general linear groups (it can also have even orthogonal factors, which we will discuss momentarily). Associated to the Langlands parameter $\varphi_{s}$ is an $L$-packet on $H$, whose stable character we denote by $S \Theta_{\varphi_{s}}$ (this is the stable form (2.2.2) in [Arthur 2013]). Let $\mathfrak{z}_{\mathfrak{e}}$ denote the pair $\left(H, \xi_{H_{1}}\right)$. This is strictly speaking not a $z$-pair in the sense of [Kottwitz and Shelstad 1999, Section 2.2], because $H$ will in general not have a simply connected derived group, but this will not cause any trouble. Let $\Delta\left[\psi, \mathfrak{e}, \mathfrak{z}_{\mathfrak{e}}\right]$ denote 
the Whittaker normalization of the Langlands-Shelstad transfer factor. Arthur shows that if $f \in \mathscr{H}(G)$ and $f^{s} \in \mathscr{H}(H)$ have $\Delta\left[\psi, \mathfrak{e}, \mathfrak{z}_{\mathfrak{e}}\right]$-matching orbital integrals, then

$$
S \Theta_{\varphi_{s}}\left(f^{s}\right)=\sum_{\rho \in \operatorname{Irr}\left(\mathscr{Y}_{\varphi}\right)}\langle s, \rho\rangle \Theta_{\pi_{\rho}}(f) .
$$

The group $\mathscr{Y}_{\varphi}$ is finite and abelian, and $\operatorname{Irr}\left(\mathscr{Y}_{\varphi}\right)$ is the set of characters of $\mathscr{S}_{\varphi}$, which is also a finite abelian group. Performing Fourier-inversion on these finite abelian groups one obtains

$$
\Theta_{\pi_{\rho}}(f)=\left|\mathscr{S}_{\varphi}\right|^{-1} \sum_{s \in \mathscr{S}_{\varphi}} \overline{\langle s, \rho\rangle} S \Theta_{\varphi^{s}}\left(f^{s}\right) .
$$

This formula is the inversion of endoscopic transfer in the $p$-adic case.

If $G$ is an even orthogonal group, the following subtle complication occurs: the group $\mathbb{Z} / 2 \mathbb{Z}$ acts on both $G$ and $\hat{G}$ by outer automorphisms, and Theorem 8.4.1 of [Arthur 2013] associates to a given tempered Langlands parameter $\varphi$ not one, but two $L$-packets $\Pi_{\varphi, 1}$ and $\Pi_{\varphi, 2}$. Each of them comes with a canonical bijection $\iota_{B, \psi, i}: \operatorname{Irr}\left(\mathscr{Y}_{\varphi}\right) \rightarrow \Pi_{\varphi, i}$, and for each $\rho \in \operatorname{Irr}\left(\mathscr{S}_{\varphi}\right)$ the two representations $\iota_{B, \psi, 1}(\rho)$ and $\iota_{B, \psi, 2}(\rho)$ are an orbit under the action of $\mathbb{Z} / 2 \mathbb{Z}$. For each $\varphi$, there is a dichotomy: either $\Pi_{\varphi, 1}=\Pi_{\varphi, 2}$, and $\mathbb{Z} / 2 \mathbb{Z}$ acts trivially on this $L$-packet; or $\Pi_{\varphi, 1} \cap \Pi_{\varphi, 2}=\varnothing$, and the action of $1 \in \mathbb{Z} / 2 \mathbb{Z}$ interchanges $\Pi_{\varphi, 1}$ and $\Pi_{\varphi, 2}$. In this situation, we will take $\iota_{B, \psi}(\rho)$ to mean the pair of representations $\left\{\iota_{B, \psi, 1}(\rho), \iota_{B, \psi, 2}(\rho)\right\}$. Following Arthur, we will use the notation $\tilde{\mathscr{H}}(G)$ to denote the subalgebra of $\mathbb{Z} / 2 \mathbb{Z}$-fixed functions in $\mathscr{H}(G)$ if $G$ is a $p$-adic even orthogonal group. For all other simple groups $G$, we set $\tilde{\mathscr{H}}(G)$ equal to $\mathscr{H}(G)$. If $G$ is a product of simple factors $G_{i}$, then $\tilde{\mathscr{H}}(G)$ is determined by $\tilde{\mathscr{H}}\left(G_{i}\right)$. All constructions, as well as the two character identities displayed above, continue to hold, but only for functions $f \in \tilde{\mathscr{H}}(G)$. Notice that on $f \in \tilde{\mathscr{H}}(G)$, the characters of the two representations $\iota_{B, \psi, 1}(\rho)$ and $\iota_{B, \psi, 2}(\rho)$ evaluate equally, and moreover $f^{s} \in \tilde{\mathscr{H}}(H)$, so the above character relations do indeed make sense.

We will now describe the analogous formulas in the real case, which are results of Shelstad [2008]. Let $G$ be a quasisplit connected reductive group defined over $F=\mathbb{R}$ and fix a Whittaker datum $(B, \psi)$. Let $\varphi: W \rightarrow{ }^{L} G$ be a tempered Langlands parameter, and $\mathscr{S}_{\varphi}$ as above. One complicating factor in the real case is that, while there is a canonical map

$$
\Pi_{\varphi} \rightarrow \operatorname{Irr}\left(\mathscr{S}_{\varphi}\right)
$$

it is not bijective, but only injective. It was observed by Adams, Barbasch, and Vogan that, in order to obtain a bijective map, one must replace $\Pi_{\varphi}$ by the disjoint union of multiple $L$-packets. All these $L$-packets correspond to $\varphi$, but belong to different inner forms of $G$. The correct inner forms to take are the ones parametrized 
by $H^{1}\left(F, G_{\mathrm{sc}}\right)$. The disjoint union of these inner forms is sometimes called the $K$-group associated to $G$, and denoted ${ }^{K} G$. For an exposition on $K$-groups we refer the reader to [Arthur 1999, Section 2] and [Shelstad 2008]. Writing $\boldsymbol{\Pi}_{\varphi}$ for the disjoint union of $L$-packets over all inner forms in the $K$-group, one now has again a bijection

$$
\Pi_{\varphi} \rightarrow \operatorname{Irr}\left(\mathscr{S}_{\varphi}\right)
$$

(see [Shelstad 2008, Section 11]) whose inverse we will denote by $\iota_{B, \psi}$, and we denote by $\langle\cdot, \cdot\rangle$ again the pairing between $\mathscr{S}_{\varphi}$ and $\boldsymbol{\Pi}_{\varphi}$ given by this bijection. Note that Shelstad uses a variant of $\mathscr{S}_{\varphi}$ involving the simply connected cover of $\hat{G}$. Since we are only considering quasisplit $K$-groups (that is, those which contain a quasisplit form), this variant will not be necessary and the group $\mathscr{S}_{\varphi}$ will be enough.

From a semisimple element $s \in \operatorname{Cent}(\varphi, \hat{G})$ we obtain an endoscopic datum $\mathfrak{e}$ by the same procedure as in the $p$-adic case just described. A second complicating factor is that, contrary to $p$-adic case discussed above, there will in general be no $L$-isomorphism $\mathscr{H} \rightarrow{ }^{L} H$. Instead, one chooses a $z$-extension $H_{1}$ of $H$. Then there exists an $L$-embedding $\xi_{H_{1}}: \mathscr{H} \rightarrow{ }^{L} H_{1}$. We let $\mathfrak{z}_{\mathfrak{e}}$ denote the datum $\left(H_{1}, \xi_{H_{1}}\right)$. Then $\varphi_{s}=\xi_{H_{1}} \circ \varphi$ is a tempered Langlands parameter for $H_{1}$ and Shelstad [2008, Section 11] shows that for any two functions $f \in \mathscr{H}\left({ }^{K} G\right)$ and $f^{s} \in \mathscr{H}\left(H_{1}\right)$ whose orbital integrals are $\Delta\left[\psi, \mathfrak{e}, \mathfrak{z}_{\mathfrak{e}}\right]$-matching, one has

and

$$
S \Theta_{\varphi_{s}}\left(f^{s}\right)=\sum_{\rho \in \operatorname{Irr}\left(\mathscr{S}_{\varphi}\right)}\langle s, \rho\rangle \Theta_{\pi_{\rho}}(\boldsymbol{f})
$$

$$
\Theta_{\pi_{\rho}}(f)=\left|\mathscr{S}_{\varphi}\right|^{-1} \sum_{s \in \mathscr{S}_{\varphi}} \overline{\langle s, \rho\rangle} S \Theta_{\varphi^{s}}\left(f^{s}\right)
$$

In the following sections, we will not use the notation ${ }^{K} G$ for a $K$-group and the boldface symbols for objects associated with it. Rather, we will treat it like a regular group and denote it by $G$, in order to simplify the statements of the results. We also note that the finite abelian groups $\mathscr{S}_{\varphi}$ occurring here are in fact 2-groups, so we may remove the complex conjugation from $\langle s, \rho\rangle$ in the inversion formulas.

\section{Change of Whittaker data}

Let $G$ be a quasisplit connected reductive group defined over a real or $p$-adic field $F$. Given a finite abelian group $A$, we will write $A^{D}$ for its group of characters. To save notation, we will write $\hat{Z}$ for the center of $\hat{G}$, and $\hat{Z}_{\mathrm{sc}}$ for the center of $\hat{G}_{\mathrm{sc}}$.

Lemma 4.1. There exists a canonical injection (bijection, if $F$ is $p$-adic)

$$
G_{\mathrm{ad}}(F) / G(F) \rightarrow \operatorname{ker}\left(H^{1}\left(W, \hat{Z}_{\mathrm{sc}}\right) \rightarrow H^{1}(W, \hat{Z})\right)^{D} .
$$


Proof. We will write $G(F)^{\tilde{D}}$ for the set of continuous characters on $G(F)$ which are trivial on the image of $G_{\mathrm{sc}}(F)$. Recall that Langlands [1989] has constructed surjective homomorphisms $H^{1}(W, \hat{Z}) \rightarrow G(F)^{\tilde{D}}$ and $H^{1}\left(W, \hat{Z}_{\mathrm{sc}}\right) \rightarrow G_{\mathrm{ad}}(F)^{\tilde{D}}$ (see [Borel 1979, Section 10] for an exposition of the construction). If $F$ is $p$-adic, they are also bijective and the statement follows right away, because the finite abelian group $G_{\mathrm{ad}}(F) / G(F)$ is Pontryagin dual to

$$
\operatorname{ker}\left(G_{\mathrm{ad}}(F)^{\tilde{D}} \rightarrow G(F)^{\tilde{D}}\right)
$$

If $F$ is real, the kernel of $H^{1}\left(W, \hat{Z}_{\mathrm{sc}}\right) \rightarrow G_{\mathrm{ad}}(F)^{\tilde{D}}$ maps onto the kernel of $H^{1}(W, \hat{Z}) \rightarrow G(F)^{\tilde{D}}$ (this is obvious from the reinterpretation of these homomorphisms given in [Kaletha 2012, Section 3.5]). This implies that the kernel of

$$
H^{1}\left(W, \hat{Z}_{\mathrm{sc}}\right) \rightarrow H^{1}(W, \hat{Z})
$$

surjects onto (3).

Let $\mathfrak{w}, \mathfrak{w}^{\prime}$ be two Whittaker data for $G$. We denote by $\left(\mathfrak{w}, \mathfrak{w}^{\prime}\right)$ the unique element of $G_{\mathrm{ad}}(F) / G(F)$ which conjugates $\mathfrak{w}$ to $\mathfrak{w}^{\prime}$. We view this element as a character on the finite abelian group

$$
\operatorname{ker}\left(H^{1}\left(W, \hat{Z}_{\mathrm{sc}}\right) \rightarrow H^{1}(W, \hat{Z})\right)
$$

via Lemma 4.1. Given a Langlands parameter $\varphi: W^{\prime} \rightarrow{ }^{L} G$, we consider the composition

$$
H^{0}(W, \varphi, \hat{G}) \rightarrow H^{0}\left(W, \varphi, \hat{G}_{\mathrm{ad}}\right) \rightarrow H^{1}\left(W, \hat{Z}_{\mathrm{sc}}\right)
$$

where $H^{0}(W, \varphi,-)$ denotes the set of invariants of $W$ with respect to the action given by $\varphi$. This map is continuous, hence it kills the connected component of the algebraic group $H^{0}(W, \varphi, \hat{G})$. Furthermore, it kills $H^{0}(W, \hat{Z})$. Thus we obtain a map

$$
\pi_{0}\left(\operatorname{Cent}(\varphi(W), \hat{G}) / Z(\hat{G})^{\Gamma}\right) \rightarrow \operatorname{ker}\left(H^{1}\left(W, \hat{Z}_{\mathrm{sc}}\right) \rightarrow H^{1}(W, \hat{Z})\right)
$$

Composing this map with the map

$$
\pi_{0}\left(S_{\varphi} / Z(\hat{G})^{\Gamma}\right) \rightarrow \pi_{0}\left(\operatorname{Cent}(\varphi(W), \hat{G}) / Z(\hat{G})^{\Gamma}\right)
$$

induced by the inclusion $S_{\varphi} \rightarrow \operatorname{Cent}(\varphi(W), \hat{G})$, we see that $\left(\mathfrak{w}, \mathfrak{w}^{\prime}\right)$ gives rise to a character on $\pi_{0}\left(S_{\varphi} / Z(\hat{G})^{\Gamma}\right)$, which we again denote by $\left(\mathfrak{w}, \mathfrak{w}^{\prime}\right)$.

Now let $s \in S_{\varphi}$. Consider the endoscopic datum $\mathfrak{e}=(H, \mathcal{H}, s, \xi)$ determined by $s$, as described in Section 3. Let $\mathfrak{z}_{\mathfrak{e}}$ be any $z$-pair for $\mathfrak{e}$. We denote by $\Delta\left[\mathfrak{w}, \mathfrak{e}, \mathfrak{z}_{\mathfrak{e}}\right]$ the Langlands-Shelstad transfer factor [Langlands and Shelstad 1987], normalized with respect to $\mathfrak{w}$ (whose definition we will briefly recall in the following proof). 


\section{Lemma 4.2.}

$$
\Delta\left[\mathfrak{w}^{\prime}, \mathfrak{e}, \mathfrak{z}_{\mathfrak{e}}\right]=\left\langle\left(\mathfrak{w}, \mathfrak{w}^{\prime}\right), s\right\rangle \cdot \Delta\left[\mathfrak{w}, \mathfrak{e}, \mathfrak{z}_{\mathfrak{e}}\right] .
$$

Proof. Write $\mathfrak{w}=(B, \psi)$. Let $\mathrm{spl}=\left(T, B,\left\{X_{\alpha}\right\}\right)$ be a splitting of $G$ containing the Borel subgroup $B$ given by $\mathfrak{w}$ and $\psi_{F}: F \rightarrow \mathbb{C}^{\times}$be a character with the property that spl and $\psi_{F}$ give rise to $\psi$ as in [Kottwitz and Shelstad 1999, Section 5.3]. Then $\Delta\left[\mathfrak{w}, \mathfrak{e}, \mathfrak{z}_{\mathfrak{e}}\right]$ is defined as the product

$$
\epsilon\left(V_{G, H}, \psi_{F}\right) \cdot \Delta\left[\mathrm{spl}, \mathfrak{e}, \mathfrak{z}_{\mathfrak{e}}\right],
$$

where $\Delta\left[\operatorname{spl}, \mathfrak{e}, \mathfrak{z}_{\mathfrak{e}}\right]$ is the normalization of the transfer factor relative to the splitting spl as constructed in [Langlands and Shelstad 1987, Section 3.7] (where it is denoted by $\left.\Delta_{0}\right)$, and $\epsilon\left(V_{G, H}, \psi_{F}\right)$ is the epsilon factor (with Langlands' normalization; see for example [Tate 1979, (3.6)]) of the degree-zero virtual $\Gamma$ representation

$$
V_{G, H}=X^{*}(T) \otimes \mathbb{C}-X^{*}\left(T^{H}\right) \otimes \mathbb{C},
$$

where $T^{H}$ is any maximally split maximal torus of $H$.

Let $g \in G_{\text {ad }}(F)$ be an element with $\operatorname{Ad}(g) \mathfrak{w}=\mathfrak{w}^{\prime}$. Put spl ${ }^{\prime}=\operatorname{Ad}(g) \operatorname{spl}$. Then $\mathrm{spl}^{\prime}$ and $\psi_{F}$ give rise to the Whittaker datum $\mathfrak{w}^{\prime}$, and consequently we have

$$
\Delta\left[\mathfrak{w}^{\prime}, \mathfrak{e}, \mathfrak{z}_{\mathfrak{e}}\right]=\epsilon\left(V_{G, H}, \psi_{F}\right) \cdot \Delta\left[\operatorname{spl}^{\prime}, \mathfrak{e}, \mathfrak{z}_{\mathfrak{e}}\right] .
$$

Let $z=g^{-1} \sigma(g) \in H^{1}\left(F, Z\left(G_{\text {sc }}\right)\right)$. Choose any maximal torus $S$ of $G$ coming from $H$ (that is, $S$ is the image of an admissible embedding into $G$ of a maximal torus of $H$ ). According to [Langlands and Shelstad 1987, Section 2.3], we have

$$
\Delta\left[\mathrm{spl}^{\prime}, \mathfrak{e}, \mathfrak{z}_{\mathfrak{e}}\right]=\langle z, s\rangle \Delta\left[\mathrm{spl}, \mathfrak{e}, \mathfrak{z}_{\mathfrak{e}}\right],
$$

where $z$ is mapped under $H^{1}\left(F, Z\left(G_{\mathrm{sc}}\right)\right) \rightarrow H^{1}\left(F, S_{\mathrm{sc}}\right)$ and $s$ is mapped under $Z(\hat{H})^{\Gamma} \rightarrow \hat{S}^{\Gamma} \rightarrow\left[\hat{S}_{\mathrm{ad}}\right]^{\Gamma}$, and the pairing uses Tate-Nakayama duality. The number $\langle z, s\rangle$ can also be obtained by mapping $s$ under

$$
H^{0}(W, Z(\hat{H})) \rightarrow H^{0}(W, \varphi, \hat{G}) \rightarrow H^{0}\left(W, \varphi, \hat{G}_{\mathrm{ad}}\right) \rightarrow H^{1}\left(W, Z\left(\hat{G}_{\mathrm{sc}}\right)\right)
$$

and pairing it with $z$, using the duality between $H^{1}\left(F, Z\left(G_{\mathrm{sc}}\right)\right)=H^{1}\left(F, S_{\mathrm{sc}} \rightarrow S_{\mathrm{ad}}\right)$ and $H^{1}\left(W, Z\left(\hat{G}_{\mathrm{sc}}\right)\right)=H^{1}\left(W, \hat{S}_{\mathrm{sc}} \rightarrow \hat{S}_{\mathrm{ad}}\right)$. Using [Kaletha 2012, Section 3.5], one sees that this is the same as the number $\left\langle\left(\mathfrak{w}, \mathfrak{w}^{\prime}\right), s\right\rangle$.

Theorem 4.3. Let $G$ be a quasisplit real $K$-group, or a quasisplit symplectic or special orthogonal $p$-adic group. For any tempered Langlands parameter $\varphi: W^{\prime} \rightarrow{ }^{L} G$ and every $\rho \in \operatorname{Irr}\left(\mathscr{S}_{\varphi}\right)$, we have

$$
\iota_{\mathfrak{w}}(\varphi, \rho)=\iota_{\mathfrak{w}}\left(\varphi, \rho \otimes\left(\mathfrak{w}, \mathfrak{w}^{\prime}\right)^{-1}\right),
$$

provided that $\iota_{\mathfrak{w}}$ and $\iota_{\mathfrak{w}^{\prime}}$ are normalized to satisfy the endoscopic character identities with respect to the transfer factors $\Delta[\mathfrak{w},-,-]$ and $\Delta\left[\mathfrak{w}^{\prime},-,-\right]$. 
Proof. Fix a semisimple $s \in S_{\varphi}$. As described in Section 3, the pair $(\varphi, s)$ gives rise to an endoscopic datum $\mathfrak{e}$, and after a choice of a $z$-pair $\mathfrak{z}_{\mathfrak{e}}=\left(H_{1}, \xi_{H_{1}}\right)$ for $\mathfrak{e}$, it further gives rise to a Langlands parameter $\varphi_{s}$ for $H_{1}$. If the functions $f \in \tilde{\mathscr{H}}(G)$ and $f^{s} \in \tilde{\mathscr{H}}\left(H_{1}\right)$ have $\Delta\left[\mathfrak{w}, \mathfrak{e}, \mathfrak{z}_{\mathfrak{e}}\right]$-matching orbital integrals, then by Lemma 4.2 the functions $f$ and $\left\langle\left(\mathfrak{w}, \mathfrak{w}^{\prime}\right), s\right\rangle \cdot f^{s}$ have $\Delta\left[\mathfrak{w}^{\prime}, \mathfrak{e}, \mathfrak{z}_{\mathfrak{e}}\right]$-matching orbital integrals. Thus

$$
\begin{aligned}
\sum_{\rho}\langle s, \rho\rangle \Theta_{\iota_{\mathfrak{w}^{\prime}}(\varphi, \rho)}(f) & =\left\langle\left(\mathfrak{w}, \mathfrak{w}^{\prime}\right), s\right\rangle S \Theta_{\varphi^{s}}\left(f^{s}\right) \\
& =\left\langle\left(\mathfrak{w}, \mathfrak{w}^{\prime}\right), s\right\rangle \sum_{\rho}\langle s, \rho\rangle \Theta_{\iota_{\mathfrak{w}}(\varphi, \rho)}(f) \\
& =\sum_{\rho}\left\langle s, \rho \otimes\left(\mathfrak{w}, \mathfrak{w}^{\prime}\right)\right\rangle \Theta_{\iota_{\mathfrak{w}}(\varphi, \rho)}(f) \\
& =\sum_{\rho}\langle s, \rho\rangle \Theta_{\iota_{\mathfrak{w}}\left(\varphi, \rho \otimes\left(\mathfrak{w}, \mathfrak{w}^{\prime}\right)^{-1}\right)}(f),
\end{aligned}
$$

where the sums run over $\rho \in \operatorname{Irr}\left(\mathscr{Y}_{\varphi}\right)$. Since this is true for all $s$, Fourier inversion gives the result.

\section{Tempered representations and their contragredient}

In this section, we will prove formula (2) for quasisplit real $K$-groups and quasisplit $p$-adic symplectic and special orthogonal groups. The bulk of the work lies in an analysis of some properties of transfer factors. We refer the reader to [Langlands and Shelstad 1987, Sections 2-3] and [Kottwitz and Shelstad 1999, Sections 3-4] for the construction of transfer factors and the associated cohomological data.

Let $F$ be $\mathbb{R}$ or a finite extension of $\mathbb{Q}_{p}$, and $G$ a quasisplit connected reductive group over $F$. We fix an $F$-splitting spl $=\left(T, B,\left\{X_{\alpha}\right\}\right)$ of $G$. We write $\hat{G}$ for the complex dual of $G$ and fix a splitting $\widehat{\mathrm{spl}}=\left(\hat{T}, \hat{B},\left\{X_{\hat{\alpha}}\right\}\right)$. We assume that the action of $\Gamma$ on $\hat{G}$ preserves $\widehat{\mathrm{spl}}$, and that there is an isomorphism $X_{*}(T) \cong X^{*}(\hat{T})$ which identifies the $B$-positive cone with the $\hat{B}$-positive cone. Let $\hat{C}$ be the Chevalley involution on $\hat{G}$ which sends $\widehat{\text { spl }}$ to the opposite splitting [Adams and Vogan 2012, Section 2]. The automorphism $\hat{C}$ commutes with the action of $\Gamma$ and thus ${ }^{L} C=\hat{C} \times \mathrm{id}_{W}$ is an $L$-automorphism of ${ }^{L} G$.

Consider a function $c: R(T, G) \rightarrow \bar{F}^{\times}$which is invariant under the action of $\Omega(T, G) \times\{ \pm 1\}$ on $R(T, G)$ and equivariant under the action of $\Gamma$. Then $\left(T, B,\left\{c_{\alpha} X_{\alpha}\right\}\right)$ is another $F$-splitting of $G$, which we will denote by $c \cdot$ spl. Given any maximal torus $S \subset G$ and any Borel subgroup $B_{S}$ containing $S$ and defined over $\bar{F}$, the admissible isomorphism $T \rightarrow S$ which sends $B$ to $B_{S}$ transports $c$ to a function $c: R(S, G) \rightarrow \bar{F}^{\times}$which is again $(\Omega(S, G) \times\{ \pm 1\})$-invariant and $\Gamma$-equivariant. Moreover, the latter function is independent of the choice of $B_{S}$ (and also of $B$ ). If $A=\left(a_{\alpha}\right)_{\alpha \in R(S, G)}$ is a set of $a$-data for $R(S, G)$, then $c \cdot A=\left(c_{\alpha} a_{\alpha}\right)_{\alpha \in R(S, G)}$ is also a set of $a$-data. 
Let $\lambda$ denote the splitting invariant constructed in [Langlands and Shelstad 1987, Section 2.3].

Lemma 5.1.

$$
\lambda(S, A, c \cdot \mathrm{spl})=\lambda(S, c \cdot A, \mathrm{spl}) .
$$

Proof. We begin by recalling the construction of the splitting invariant. For a simple root $\alpha \in R(T, G)$, let $\eta_{\alpha}^{\mathrm{spl}}: \mathrm{SL}_{2} \rightarrow G$ be the homomorphism determined by the splitting spl. We put

$$
n_{\mathrm{spl}}\left(s_{\alpha}\right)=\eta_{\alpha}^{\mathrm{spl}}\left(\begin{array}{rr}
0 & 1 \\
-1 & 0
\end{array}\right)
$$

For any $w \in \Omega(T, G)$ choose a reduced expression $w=s_{\alpha_{1}} \cdots s_{\alpha_{n}}$ and set

$$
n_{\mathrm{spl}}(w)=n_{\mathrm{spl}}\left(s_{\alpha_{1}}\right) \cdots n_{\mathrm{spl}}\left(s_{\alpha_{n}}\right) .
$$

By [Springer 1981, Section 11.2.9], this product is independent of the choice of reduced expression.

We choose a Borel subgroup $B_{S} \subset G$ defined over $\bar{F}$ and containing $S$, and an element $h \in G(\bar{F})$ such that $\operatorname{Ad}(h)(T, B)=\left(S, B_{S}\right)$. Then, for $\sigma \in \Gamma$ and $s \in S$, we have

$$
\operatorname{Ad}\left(h^{-1}\right)\left[{ }^{\sigma} s\right]={ }^{w_{S}(\sigma) \sigma} \operatorname{Ad}\left(h^{-1}\right)[s]
$$

for some $w_{S}(\sigma) \in \Omega(T, G)$. Then $\lambda(S, A, \operatorname{spl}) \in H^{1}(F, S)$ is the element whose image under $\operatorname{Ad}\left(h^{-1}\right)$ is represented by the cocycle

$$
\sigma \mapsto \prod_{\substack{\alpha>0 \\\left(w_{S}(\sigma) \sigma\right)^{-1} \alpha<0}} \alpha^{\vee}\left(a_{\mathrm{Ad}(h) \alpha}\right) \cdot n_{\mathrm{spl}}\left(w_{S}(\sigma)\right) \cdot \sigma\left(h^{-1}\right) h,
$$

where $\alpha>0$ means that $\alpha \in R(T, G)$ is $B$-positive.

We now examine the relationship between $n_{\text {spl }}$ and $n_{c \cdot \text { spl }}$. Recall the standard triple $(E, H, F)$ in $\operatorname{Lie}\left(\mathrm{SL}_{2}\right)$, where

$$
E=\left[\begin{array}{ll}
0 & 1 \\
0 & 0
\end{array}\right], \quad H=\left[\begin{array}{rr}
1 & 0 \\
0 & -1
\end{array}\right], \quad \text { and } \quad F=\left[\begin{array}{ll}
0 & 0 \\
1 & 0
\end{array}\right] .
$$

The differential $d \eta_{\alpha}^{\text {spl }}$ sends $(E, H, F)$ to $\left(X_{\alpha}, H_{\alpha}, X_{-\alpha}\right)$, where $H_{\alpha}=d \alpha^{\vee}(1)$ and $X_{-\alpha} \in \mathfrak{g}_{-\alpha}$ is determined by $\left[X_{\alpha}, X_{-\alpha}\right]=H_{\alpha}$. On the other hand, the differential $d \eta_{\alpha}^{c \text { spl }}$ sends $(E, H, F)$ to $\left(c_{\alpha} \cdot X_{\alpha}, H_{\alpha}, c_{\alpha}^{-1} X_{-\alpha}\right)$. Thus

$$
\eta_{\alpha}^{c \cdot s p l}=\eta_{\alpha}^{\mathrm{spl}} \circ \operatorname{Ad}\left[\begin{array}{cc}
\sqrt{c_{\alpha}} & 0 \\
0 & \sqrt{c_{\alpha}}-1
\end{array}\right]
$$

for an arbitrary choice of a square root of $c_{\alpha}$. It follows that

$$
n_{c \cdot \mathrm{spl}}\left(s_{\alpha}\right)=\alpha^{\vee}\left(c_{\alpha}\right) \cdot n_{\mathrm{spl}}\left(s_{\alpha}\right) .
$$


Using induction and [Bourbaki 2002, Chapter VI, Section 1.6, Corollary 2], we conclude that for any $w \in \Omega(T, G)$, we have

$$
n_{c \cdot \mathrm{spl}}(w)=\prod_{\substack{\alpha>0 \\ w^{-1} \alpha<0}} \alpha^{\vee}\left(c_{\alpha}\right) \cdot n_{\mathrm{spl}}(w) .
$$

From this equation and the fact that any $\sigma \in \Gamma$ preserves the sets of $B$-positive and $B$-negative roots, we see that

$$
n_{c \cdot \mathrm{spl}}\left(w_{S}(\sigma)\right)=\prod_{\substack{\alpha>0 \\\left(w_{S}(\sigma) \sigma\right)^{-1} \alpha<0}} \alpha^{\vee}\left(c_{\alpha}\right) \cdot n_{\mathrm{spl}}\left(w_{S}(\sigma)\right) .
$$

The statement follows by comparing (5) for $\lambda(S, A, c \cdot \mathrm{spl})$ and $\lambda(S, c \cdot A, \mathrm{spl})$.

Given a torus $S$ defined over $F$, we will denote by -1 the homomorphism $S \rightarrow S$ which sends $s \in S$ to $s^{-1}$. It is of course defined over $F$. Its dual ${ }^{L} S \rightarrow{ }^{L} S$ is given by $(s, w) \mapsto\left(s^{-1}, w\right)$ and will also be denoted by -1 . Given a maximal torus $S \subset G$ and a set of $\chi$-data $X=\left\{\chi_{\alpha} \mid \alpha \in R(S, G)\right\}$ for $R(S, G)$, we denote by $-X$ the set $\left\{\chi_{\alpha}^{-1} \mid \alpha \in R(S, G)\right\}$. This is also a set of $\chi$-data. It is shown in [Langlands and Shelstad 1987, Section 2.6] that $X$ provides a canonical $\hat{G}$-conjugacy class of $L$-embeddings ${ }^{L} \xi_{X}:{ }^{L} S \rightarrow{ }^{L} G$.

Lemma 5.2. Let $S \subset G$ be a maximal torus defined over $F$, and let $X$ be $\chi$-data for $R(S, G)$. Let ${ }^{L} \xi_{X}:{ }^{L} S \rightarrow{ }^{L} G$ be the canonical $\hat{G}$-conjugacy class of embeddings associated to $X$. Then the $\hat{G}$-conjugacy classes of maps ${ }^{L} \xi_{X} \circ(-1)$ and ${ }^{L} C \circ{ }^{L} \xi_{-X}$ are equal.

Remark. Intuitively, we can express the statement of Lemma 5.2 by the following diagram, whose commutativity is to be understood up to $\hat{G}$-conjugacy:

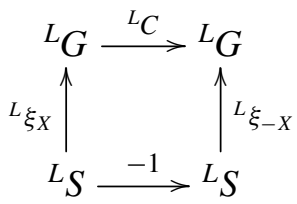

Proof. We choose a representative ${ }^{L} \xi_{X}$ within its $\hat{G}$-conjugacy class by following the constructions in [Langlands and Shelstad 1987, Section 2.6]. For this, we choose a Borel subgroup defined over $\bar{F}$ and containing $S$. This provides an admissible isomorphism $\hat{\xi}: \hat{S} \rightarrow \hat{T}$. For $w \in W$, let $\sigma_{S}(w) \in \Omega(\hat{T}, \hat{G})$ be defined by

$$
\hat{\xi}\left({ }^{w_{S}}\right)=\sigma_{S}(w) w \hat{\xi}(s) .
$$

Then a representative of ${ }^{L} \xi_{X}$ is given by

$$
L_{\xi_{X}}(s, w)=\left[\hat{\xi}(s) r_{\hat{B}, X}(w) n_{\widehat{s p l}}\left(\sigma_{S}(w)\right), w\right],
$$


where $r_{\hat{B}, X}$ denotes the cochain $r_{p}$ constructed in [Langlands and Shelstad 1987, Section 2.6] from the $\chi$-data $X$ and the gauge $p$ determined by $\hat{B}$, and $n_{\widehat{\mathrm{spl}}}$ is the section $\Omega(\hat{T}, \hat{G}) \rightarrow N(\hat{T}, \hat{G})$ determined by the splitting $\widehat{\text { spl }}$ as described in the proof of the previous lemma. Using the fact that $\hat{C}$ acts by inversion on $\hat{T}$ and [Adams and Vogan 2012, Lemma 5.8], we see that

$$
{ }^{L} C \circ{ }^{L} \xi_{X}(s, w)=\left[\hat{\xi}(s)^{-1} r_{\hat{B}, X}(w)^{-1} n_{\widehat{s p l}}\left(\sigma_{S}(w)^{-1}\right)^{-1}, w\right] .
$$

One sees that $r_{\hat{B}, X}(w)^{-1}=r_{\hat{B},-X}(w)$. Moreover, by [Adams and Vogan 2012, Lemma 5.4] we have

$$
n_{\mathrm{spl}}\left(\sigma_{S}(w)^{-1}\right)^{-1}=\left[t \cdot \sigma_{S}(w) t^{-1}\right] n_{\mathrm{spl}}\left(\sigma_{S}(w)\right),
$$

where $t \in \hat{T}$ is any lift of $\rho^{\vee}(-1) \in \hat{T}_{\text {ad }}, \rho^{\vee}$ being half the sum of the positive coroots. We can choose $t \in \hat{T}^{\Gamma}$ by choosing a root $i$ of -1 and putting $t=\prod_{\alpha \in R(\hat{T}, \hat{B})} \alpha^{\vee}(i)$. Then we see that $\alpha \in R(\hat{T}, \hat{B})$

$$
{ }^{L} C \circ{ }^{L} \xi_{X}(s, w)=\operatorname{Ad}(t) \circ{ }^{L} \xi_{-X} \circ(-1)(s, w) .
$$

Let $\theta$ be an automorphism which preserves spl, and let $\boldsymbol{a} \in H^{1}(W, Z(\hat{G}))$. The class $\boldsymbol{a}$ corresponds to a character $\omega: G(F) \rightarrow \mathbb{C}^{\times}$. Let $\hat{\theta}$ be the automorphism dual to $\theta$, which preserves $\widehat{\text { spl}}$. Note that $\hat{\theta}$ commutes with the action of $\Gamma$. We will write ${ }^{L} \theta$ for $\theta \times \operatorname{id}_{W}$.

Let us recall some basic facts from [Kottwitz and Shelstad 1999, Section 1]. Let $\hat{G}^{1}$ be the connected component of the group $\hat{G}^{\hat{\theta}}$, let $\hat{T}^{1}=\hat{T} \cap \hat{G}^{1}$, and let $\hat{B}^{1}=\hat{B} \cap \hat{G}^{1}$. Then $\hat{G}^{1}$ is a reductive group and $\left(\hat{T}^{1}, \hat{B}^{1}\right)$ is a Borel pair for it. The set $\Delta\left(\hat{T}^{1}, \hat{B}^{1}\right)$ of $\hat{B}^{1}$-simple roots for $\hat{T}^{1}$ is the set of restrictions to $\hat{T}^{1}$ of the set $\Delta(\hat{T}, \hat{B})$ of $\hat{B}$-simple roots for $\hat{T}$. Moreover, the fibers of the restriction map

$$
\text { res }: \Delta(\hat{T}, \hat{B}) \rightarrow \Delta\left(\hat{T}^{1}, \hat{B}^{1}\right)
$$

are precisely the $\langle\hat{\theta}\rangle$-orbits in $\Delta(\hat{T}, \hat{B})$. We denote the image of $\alpha$ under res by $\alpha_{\text {res. }}$.

We can extend the pair $\left(\hat{T}^{1}, \hat{B}^{1}\right)$ to a $\Gamma$-splitting $\widehat{\operatorname{spl}}^{1}=\left(\hat{T}^{1}, \hat{B}^{1},\left\{X_{\alpha_{\text {res }}}\right\}\right)$ of $\hat{G}^{1}$ by setting for each $\alpha_{\text {res }} \in \Delta\left(\hat{T}^{1}, \hat{B}^{1}\right)$

$$
X_{\alpha_{\mathrm{res}}}=\sum_{\substack{\beta \in \Delta(\hat{T}, \hat{B}) \\ \beta_{\mathrm{res}}=\alpha_{\mathrm{res}}}} X_{\beta} .
$$

Since $\hat{\theta}$ commutes with $\Gamma$, the group $\hat{G}^{1}$ and the splitting just constructed is preserved by $\Gamma$. Thus, $\hat{G}^{1} \rtimes W$ is the $L$-group of a connected reductive group $G^{1}$. Moreover, since $\hat{\theta}$ also commutes with $\hat{C}$, the automorphism $\hat{C}$ preserves the group $\hat{G}^{1}$ and acts by inversion on its maximal torus $\hat{T}^{1}$. Thus, $\hat{C}$ is a Chevalley involution for $\hat{G}^{1}$. 
Although it will not concern us, we want to remark that it is in general not true that $\hat{C}$ sends the splitting $\widehat{\mathrm{spl}}^{1}$ to its opposite. Rather, it sends $\widehat{\mathrm{spl}}^{1}$ to the splitting of $\hat{G}^{1}$ constructed from the opposite of $\widehat{\mathrm{spl}}$ by the same procedure as above. That this splitting differs from the opposite of $\widehat{\mathrm{spl}}^{1}$ is due to the fact that for $\alpha_{\text {res }} \in R\left(\hat{T}^{1}, \hat{G}^{1}\right)$, the coroot $H_{\alpha_{\mathrm{res}}}$ is not always the sum of $H_{\beta}$ for all $\beta$ in the $\Gamma$-orbit corresponding to $\alpha_{\text {res. }}$. In fact, we have

$$
H_{\alpha_{\mathrm{res}}}=c_{\alpha_{\mathrm{res}}} \cdot \sum_{\substack{\beta \in \Delta(\hat{T}, \hat{B}) \\ \beta_{\mathrm{res}}=\alpha_{\mathrm{res}}}} H_{\beta},
$$

where $c_{\alpha}=1$ if $\alpha_{\text {res }}$ is of type $R_{1}$ and $c_{\alpha}=2$ if $\alpha_{\text {res }}$ is of type $R_{2}$.

Let $\mathfrak{e}=(H, s, \mathcal{H}, \xi)$ be an endoscopic datum for $(G, \theta, \boldsymbol{a})$. Let $\left(\hat{T}^{H}, \hat{B}^{H},\left\{\hat{X}_{\beta}^{H}\right\}\right)$ be a $\Gamma$-fixed splitting of $\hat{H}$, and denote by $\hat{C}^{H}$ the corresponding Chevalley involution of $\hat{H}$ and by ${ }^{L} C^{H}$ the corresponding $L$-automorphism of ${ }^{L} H$. Let $\mathfrak{z}_{\mathfrak{e}}=\left(H_{1}, \xi_{H_{1}}\right)$ be a $z$-pair for $\mathfrak{e}$. The splitting of $\hat{H}$ provides a unique one for $\hat{H}_{1}$ and the involutions $\hat{C}^{H_{1}}$ and ${ }^{L} C^{H_{1}}$ restricted to $\hat{H}$ and ${ }^{L} H$ equal $\hat{C}^{H}$ and ${ }^{L} C^{H}$.

We write ${ }^{L} C(\mathfrak{e})$ for the quadruple $\left(H, s^{\prime}, \mathscr{H}^{\prime}, \xi^{\prime}\right)$, where $s^{\prime}=\hat{C}\left(s^{-1}\right), \mathscr{H}^{\prime}$ is the same group as $\mathscr{H}$ but with the embedding $\hat{H} \rightarrow \mathscr{H}$ composed with $\hat{C}^{H}$, and $\xi^{\prime}={ }^{L} C \circ{ }^{L} \theta \circ \xi$. We write ${ }^{L} C^{H}\left(\mathfrak{z}_{\mathfrak{e}}\right)$ for the pair $\left(H_{1},{ }^{L} C^{H_{1}} \circ \xi_{H_{1}}\right)$.

Fact 5.3. The quadruple ${ }^{L} C(\mathfrak{e})$ is an endoscopic datum for $\left(G, \theta^{-1}, \boldsymbol{a}\right)$, and ${ }^{L} C^{H}\left(\mathfrak{z}_{\mathfrak{e}}\right)$ is a $z$-pair for it. If $\mathfrak{e}^{\prime}$ is an endoscopic datum for $(G, \theta, \boldsymbol{a})$ equivalent to $\mathfrak{e}$, then ${ }^{L} C\left(\mathfrak{e}^{\prime}\right)$ is equivalent to ${ }^{L} C(\mathfrak{e})$. An isomorphism $S^{H} \rightarrow S_{\theta}$ from a maximal torus of $H$ to a maximal torus of $G^{1}$ is $\mathfrak{e}$-admissible if and only if it is ${ }^{L} C(\mathfrak{e})$-admissible.

Proof. The proof is straightforward, but we include it for the convenience of the reader. We need to check [Kottwitz and Shelstad 1999, (2.1.1-2.1.4b)]. It is clear that $\mathscr{H}^{\prime}$ remains a split extension of $\hat{H}$ by $W$. To check that $s^{\prime}$ is $\hat{\theta}^{-1}$-quasisemisimple, one observes that the automorphism $\operatorname{Int}\left(\hat{C}\left(s^{-1}\right)\right) \circ \hat{\theta}^{-1}$ is conjugate to $(\operatorname{Int}(s) \hat{\theta})^{-1}$ by $\hat{C} \circ \operatorname{Int}\left(s^{-1}\right)$. The fact that $\xi^{\prime}$ is an isomorphism onto its image is inherited from $\xi$, and the image is

$$
\begin{aligned}
\hat{C}(\hat{\theta}(\xi(\hat{H}))) & =\hat{C}\left(\hat{\theta}\left(\operatorname{Cent}(\operatorname{Int}(s) \circ \hat{\theta}, \hat{G})^{\circ}\right)\right) \\
& =\hat{C}\left(\hat{\theta}\left(\operatorname{Cent}\left(\hat{\theta}^{-1} \circ \operatorname{Int}\left(s^{-1}\right), \hat{G}\right)^{\circ}\right)\right) \\
& =\operatorname{Cent}\left(\operatorname{Int}\left(s^{\prime}\right) \circ \hat{\theta}^{-1}, \hat{G}\right)^{\circ} .
\end{aligned}
$$

Finally, we have

$$
\begin{aligned}
\operatorname{Int}\left(s^{\prime}\right) \circ{ }^{L} \theta^{-1} \circ \xi^{\prime} & ={ }^{L} C \circ \operatorname{Int}\left(s^{-1}\right) \circ \xi \\
& ={ }^{L} C \circ\left(\left(a^{\prime}\right)^{-1} \cdot{ }^{L} \theta \circ \xi\right) \\
& =a^{\prime} \cdot \xi^{\prime} .
\end{aligned}
$$


This shows that ${ }^{L} C(\mathfrak{e})$ is indeed an endoscopic datum for $\left(G, \theta^{-1}, \boldsymbol{a}\right)$. A direct computation shows that if $g \in \hat{G}$ is an isomorphism $\mathfrak{e} \rightarrow \mathfrak{e}^{\prime}$, then $\hat{C} \hat{\theta}(g)$ is an isomorphism ${ }^{L} C(\mathfrak{e}) \rightarrow{ }^{L} C\left(\mathfrak{e}^{\prime}\right)$. To check that ${ }^{L} C^{H}\left(\mathfrak{z}_{\mathfrak{e}}\right)$ is a $z$-pair for ${ }^{L} C(\mathfrak{e})$, we only need to observe that, since the restriction of ${ }^{L} C^{H_{1}}$ to $\hat{H}$ equals $\hat{C}^{H}$, the composition of ${ }^{L} C^{H_{1}} \circ \xi_{H_{1}}: \mathscr{H}_{1} \rightarrow{ }^{L_{H}} H_{1}$ with the inclusion $\hat{H} \rightarrow \mathscr{H}_{1}$ is indeed the natural inclusion $\hat{H} \rightarrow \hat{H}_{1} \rightarrow{ }^{L} H_{1}$. To compare the notions of admissible isomorphisms, replace $\mathfrak{e}$ by an equivalent datum so that $s \in \hat{T}$ and $\xi\left(\hat{T}^{H}\right) \subset \hat{T}$. Then $\xi$ restricts to an isomorphism $\hat{T}^{H} \rightarrow \hat{T}^{1}$. Recalling the definitions of $\mathscr{H}^{\prime}$ and $\xi^{\prime}$, we see that $\xi^{\prime}$ restricts to the same isomorphism. Thus the notion of admissibility of isomorphisms of tori remains unchanged when we pass from $\mathfrak{e}$ to ${ }^{L} C(\mathfrak{e})$.

Assume now that spl is $\theta$-stable and augment $B$ to a $\theta$-stable Whittaker datum $(B, \psi)$. Then, associated to $(G, \theta, \boldsymbol{a}),(B, \psi), \mathfrak{e}$, and $\mathfrak{z}_{\mathfrak{e}}$, we have the Whittaker normalization of the transfer factor for $G$ and $H_{1}$. In fact, as explained in [Kottwitz and Shelstad 2012], there are two different such normalizations - one adapted to the classical local Langlands correspondence for tori [ibid., (5.5.2)], and one adapted to the renormalized correspondence [ibid., (5.5.1)]. To be consistent with their notation, we will call these transfer factors $\Delta^{\prime}\left[\psi, \mathfrak{e}, \mathfrak{z}_{\mathfrak{e}}\right]$ (for the classical local Langlands correspondence), and $\Delta_{D}\left[\psi, \mathfrak{e}, \mathfrak{z}_{\mathfrak{e}}\right]$ (for the renormalized correspondence). On the other hand, associated to $\left(G, \theta^{-1}, \boldsymbol{a}\right),\left(B, \psi^{-1}\right),{ }^{L} C(\mathfrak{e})$, and ${ }^{L} C^{H}\left(\mathfrak{z}_{\mathfrak{e}}\right)$, we also have the Whittaker normalization of the transfer factor, again in the two versions. We will call these $\Delta^{\prime}\left[\psi^{-1},{ }^{L} C(\mathfrak{e}),{ }^{L} C^{H}\left(\mathfrak{z}_{\mathfrak{e}}\right)\right]$ and $\Delta_{D}\left[\psi^{-1},{ }^{L} C(\mathfrak{e}),{ }^{L} C^{H}\left(\mathfrak{z}_{\mathfrak{e}}\right)\right]$. In the case $\theta=1$ and $\boldsymbol{a}=1$ (that is, ordinary endoscopy), one also has the normalizations $\Delta$ and $\Delta_{D}^{\prime}$ [Section 5.1]. The normalization $\Delta$ is the one compatible with [Langlands and Shelstad 1987].

Proposition 5.4. Let $\gamma_{1} \in H_{1}(F)$ be a strongly $G$-regular semisimple element, and let $\delta \in G(F)$ be a strongly $\theta$-regular $\theta$-semisimple element. We have

$$
\Delta^{\prime}\left[\psi, \mathfrak{e}, \mathfrak{z}_{\mathfrak{e}}\right]\left(\gamma_{1}, \delta\right)=\Delta^{\prime}\left[\psi^{-1},{ }^{L} C(\mathfrak{e}),{ }^{L} C^{H}\left(\mathfrak{z}_{\mathfrak{e}}\right)\right]\left(\gamma_{1}^{-1}, \theta^{-1}\left(\delta^{-1}\right)\right) .
$$

The same equality holds with $\Delta_{D}$ in place of $\Delta^{\prime}$. Moreover, in the setting of ordinary endoscopy, the equality also holds for $\Delta$ and $\Delta_{D}^{\prime}$.

Proof. Let us first discuss the different versions of the transfer factor. In ordinary endoscopy, one obtains $\Delta$ from $\Delta^{\prime}$ by replacing $s$ with $s^{-1}$. Thus it is clear that the above equality will hold for the one if and only if it holds for the other. The same is true for $\Delta_{D}$ and $\Delta_{D}^{\prime}$. Returning to twisted endoscopy, the difference between $\Delta^{\prime}$ and $\Delta_{D}$ is more subtle, and the statement for the one does not formally follow from the statement for the other. However, the proof for both cases is the same, and we will give it for the case of $\Delta_{D}$.

One sees easily that $\gamma$ is a $\theta$-norm of $\delta$ precisely when $\gamma^{-1}$ is a $\theta^{-1}$-norm of $\theta^{-1}\left(\delta^{-1}\right)$. We assume that this is the case. Let $S_{H_{1}} \subset H_{1}$ be the centralizer of 
$\gamma_{1}$, let $S_{H} \subset H$ be the image of $S_{H_{1}}$. The torus $S_{H}$ is the centralizer of the image $\gamma \in H(F)$ of $\gamma_{1}$. We choose a $\theta$-admissible maximal torus $S \subset G$, an admissible isomorphism $\varphi: S_{H} \rightarrow S_{\theta}$, an element $\delta^{*} \in S(\bar{F})$ whose image in $S_{\theta}$ equals $\varphi(\gamma)$, and an element $g \in G_{\mathrm{sc}}(\bar{F})$ with $\delta^{*}=g \delta \theta\left(g^{-1}\right)$. The objects $\varphi, g$ and $\delta^{*}$ will enter into the construction of the transfer factor $\Delta_{D}\left[\psi, \mathfrak{e}, \mathfrak{z}_{\mathfrak{e}}\right]\left(\gamma_{1}, \delta\right)$.

As already remarked, $\gamma^{-1}$ is a $\theta^{-1}$-norm for $\theta^{-1}\left(\delta^{-1}\right)$. By Fact 5.3, $\varphi$ is an ${ }^{L} C(\mathfrak{e})$-admissible isomorphism of tori, and it is clear that $\varphi\left(\gamma^{-1}\right)$ equals the image of $\theta^{-1}\left(\delta^{-1}\right)$ in $S_{\theta}$. Moreover $\theta^{-1}\left(\delta^{*-1}\right)=g \cdot \theta^{-1}\left(\delta^{-1}\right) \cdot \theta^{-1}\left(g^{-1}\right)$. Thus we may use the objects $\varphi, g$ and $\theta^{-1}\left(\delta^{*-1}\right)$ when constructing the transfer factor $\Delta_{D}\left[\psi^{-1},{ }^{L} C(\mathfrak{e}),{ }^{L} C^{H}\left(\mathfrak{z}_{\mathfrak{e}}\right)\right]\left(\gamma_{1}^{-1}, \theta^{-1}\left(\delta^{-1}\right)\right)$.

We will also need $\theta$-invariant $a$-data A for $R(S, G)$ and $\chi$-data $\mathrm{X}$ for $R_{\text {res }}(S, G)$. Moreover, we fix an additive character $\psi_{F}: F \rightarrow \mathbb{C}^{\times}$and assume that the splitting spl $=\left(T, B, X_{\alpha}\right)$ and the character $\psi_{F}$ give rise to the fixed Whittaker datum $(B, \psi)$. Up to equivalence of endoscopic data we may assume $s \in \hat{T}$. This implies $\hat{C}\left(s^{-1}\right)=s$. Then, by [Kottwitz and Shelstad 2012, Equation 5.5.1], we have

$$
\Delta_{D}\left[\psi, \mathfrak{e}, \mathfrak{z}_{\mathfrak{e}}\right]=\epsilon\left(V_{G, H}, \psi_{F}\right) \cdot \Delta_{\mathrm{I}}^{\mathrm{new}}[\mathrm{spl}, A] \cdot \Delta_{\mathrm{II}}^{-1}[A, X] \cdot \Delta_{\mathrm{III}}\left[\mathfrak{e}, \mathfrak{z}_{\mathfrak{e}}, X\right] \cdot \Delta_{\mathrm{IV}} .
$$

The factor $\epsilon\left(V_{G, H}, \psi_{F}\right)$ is the epsilon factor (with Langlands' normalization; see for example [Tate 1979, (3.6)]) for the virtual $\Gamma$-representation

$$
V_{G, H}=X^{*}(T)^{\theta} \otimes \mathbb{C}-X^{*}\left(T^{H}\right) \otimes \mathbb{C},
$$

where $T^{H}$ is any maximally split maximal torus of $H$. It does not depend on any further data and is thus the same for both sides of the equality we are proving. One also sees immediately from the definition that

$$
\Delta_{\mathrm{IV}}\left(\gamma_{1}^{-1}, \theta^{-1}\left(\delta^{-1}\right)\right)=\Delta_{\mathrm{IV}}\left(\gamma_{1}, \delta\right) .
$$

We now examine the factors $\Delta_{\mathrm{I}}, \Delta_{\mathrm{II}}$ and $\Delta_{\mathrm{III}}$, the latter requiring the bulk of the work. These factors depend on most of the objects chosen so far. We have indicated in brackets the more important objects on which they depend, as it will be necessary to keep track of them. These are not all the dependencies. For example, all factors $\Delta_{i}$ depend on the datum $\mathfrak{e}$, but except for $\Delta_{\text {III }}$, this dependence is only through the datum $s$, which we have arranged to be equal for $\mathfrak{e}$ and ${ }^{L} C(\mathfrak{e})$, and so we have not included $\mathfrak{e}$ in the notation for these factors.

The factor $\Delta_{\mathrm{I}}^{\text {new }}[\mathfrak{e}, \mathrm{spl}, A]$ does not depend directly on $\gamma_{1}$ and $\delta$, but rather only on the choices of $S$ and $\varphi$. As we have remarked in the preceding paragraphs, these choices also serve $\gamma_{1}^{-1}$ and $\theta^{-1}\left(\delta^{-1}\right)$, and we see that

$$
\Delta_{\mathrm{I}}^{\text {new }}[\operatorname{spl}, A]\left(\gamma_{1}^{-1}, \theta^{-1}\left(\delta^{-1}\right)\right)=\Delta_{\mathrm{I}}^{\text {new }}[\operatorname{spl}, A]\left(\gamma_{1}, \delta\right) .
$$

We turn to $\Delta_{\mathrm{II}}[A, X]$. Let $-A$ denote the $a$-data obtained from $A$ by replacing each $a_{\alpha}$ by $-a_{\alpha}$. Let $-X$ denote the $\chi$-data obtained from $X$ by replacing each $\chi_{\alpha}$ by 
$\chi_{\alpha}^{-1}$. Then one checks that

$$
\Delta_{\mathrm{II}}[A, X]\left(\gamma_{1}^{-1}, \theta^{-1}\left(\delta^{-1}\right)\right)=\Delta_{\mathrm{II}}[-A,-X]\left(\gamma_{1}, \delta\right) .
$$

Before we can examine $\Delta_{\text {III }}\left[\mathfrak{e}, \mathfrak{z}_{\mathfrak{e}}, X\right]$, we need to recall its construction, following [Kottwitz and Shelstad 1999, Section 4.4 and Section 5.3]. We define an $F$-torus $S_{1}$ as the fiber product

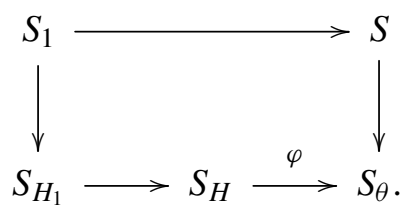

The element $\delta_{1}^{*}=\left(\gamma_{1}, \delta^{*}\right)$ belongs to $S_{1}$. The automorphism id $\times \theta$ of $S_{H_{1}} \times S$ induces an automorphism $\theta_{1}$ of $S_{1}$. This automorphism restricts trivially to the kernel of $S_{1} \rightarrow S$, and hence $1-\theta_{1}$ induces a homomorphism $S \rightarrow S_{1}$, which we can compose with $S_{\mathrm{sc}} \rightarrow S$ to obtain a homomorphism $S_{\mathrm{sc}} \rightarrow S_{1}$, which we still denote by $1-\theta_{1}$.

The element $\left(\sigma(g) g^{-1}, \delta_{1}^{*}\right)$ belongs to $H^{1}\left(F, S_{\mathrm{sc}} \stackrel{1-\theta_{1}}{\longrightarrow} S_{1}\right)$ and is called $\operatorname{inv}\left(\gamma_{1}, \delta\right)$. Kottwitz and Shelstad [1999, A.3] construct a pairing $\langle\cdot, \cdot\rangle_{\mathrm{KS}}$ between the abelian groups

$$
H^{1}\left(F, S_{\mathrm{sc}} \stackrel{1-\theta_{1}}{\longrightarrow} S_{1}\right) \text { and } H^{1}\left(W, \hat{S}_{1} \stackrel{1-\hat{\theta}_{1}}{\longrightarrow} \hat{S}_{\mathrm{ad}}\right)
$$

Using this pairing, they define

$$
\Delta_{\mathrm{III}}\left[\mathfrak{e}, \mathfrak{z}_{\mathfrak{e}}, X\right]\left(\gamma_{1}, \delta\right)=\left\langle\operatorname{inv}\left(\gamma_{1}, \delta\right), A_{0}\left[\mathfrak{e}, \mathfrak{z}_{\mathfrak{e}}, X\right]\right\rangle_{\mathrm{KS}},
$$

where $A_{0}\left[\mathfrak{e}, \mathfrak{z}_{\mathfrak{e}}, X\right]$ is an element of $H^{1}\left(F, \hat{S}_{1} \stackrel{1-\hat{\theta}_{1}}{\longrightarrow} \hat{S}_{\mathrm{ad}}\right)$ constructed as follows:

The $\chi$-data $X$ provides an $\hat{H}$-conjugacy class of embeddings ${ }^{L} S_{H} \rightarrow{ }^{L} H$ and a $\hat{G}^{1}$-conjugacy class of embeddings ${ }^{L} S_{\theta} \rightarrow{ }^{L} G^{1}$, where $\hat{G}^{1}$ is the connected stabilizer of $\hat{\theta}$. Conjugating within $\hat{H}$ and $\hat{G}^{1}$ we arrange that these embeddings map $\hat{S}_{H}$ to $\hat{T}^{H}$ and $\hat{S}_{\theta}$ to $\hat{T}^{1}$. Composing with the canonical embeddings ${ }^{L} H \rightarrow{ }^{L} H_{1}$ and ${ }^{L} G^{1} \rightarrow{ }^{L} G$, we obtain embeddings $\xi_{1}[X]:{ }^{L} S_{\theta} \rightarrow{ }^{L} G$ and $\xi_{S_{H}}[X]:{ }^{L} S_{H} \rightarrow{ }^{L} H_{1}$. There is a unique embedding $\xi_{1}^{1}[X]:{ }^{L} S \rightarrow{ }^{L} G$ extending $\xi_{1}[X]$, and there is a unique embedding $\xi_{S_{H}}^{1}[X]:{ }^{L} S_{H_{1}} \rightarrow{ }^{L} H_{1}$ extending $\xi_{S_{H}}[X]$.

Letting $\bar{x}$ denote the image of $x \in \mathscr{H}$ under the projection $\mathscr{H} \rightarrow W$, define

$$
u=\left\{x \in \mathscr{H}|\operatorname{Ad}(\xi(x))|_{\hat{T}^{1}}=\left.\operatorname{Ad}\left(\xi_{1}[X](1 \times \bar{x})\right)\right|_{\hat{T}^{1}}\right\} .
$$

Then $U$ is an extension of $W$ by $\hat{T}^{H}$. One can show that $\xi(u) \subset \xi_{1}^{1}[X]\left({ }^{L} S\right)$ and $\xi_{H_{1}}(U) \subset \xi_{S_{H}}^{1}[X]\left({ }^{L} S_{H_{1}}\right)$. Then we can define, for any $w \in W$, an element $a_{S}[X](w) \in \hat{S}_{1}$, by choosing a lift $u(w) \in \mathcal{U}$ and letting $a_{S}[X](w)=\left(t_{1}^{-1}, t\right) \in$ $\hat{S}_{H_{1}} \times \hat{S} \rightarrow \hat{S}_{1}$, where $t_{1} \in \hat{S}_{H_{1}}$ and $t \in \hat{S}$ are the unique elements satisfying

$$
\xi_{1}^{1}[X](t \times w)=\xi(u(w)) \text { and } \xi_{S_{H}}^{1}[X]\left(t_{1} \times w\right)=\xi_{H_{1}}(u(w)) .
$$


We can further define $s_{S}=\left[\xi_{1}^{1}\right]^{-1}(s) \in \hat{S}$ and also view it as an element of $\hat{S}_{\text {ad }}$. Then

$$
A_{0}\left[\mathfrak{e}, \mathfrak{z}_{\mathfrak{e}}, X\right]=\left(a_{S}[X]^{-1}, s_{S}\right) \in H^{1}\left(W, \hat{S}_{1} \rightarrow \hat{S}_{\mathrm{ad}}\right)
$$

We are now ready to examine $\Delta_{\text {III }}\left[\mathfrak{e}, \mathfrak{z}_{\mathfrak{e}}, X\right]$. We have

$$
\operatorname{inv}\left(\gamma_{1}^{-1}, \theta^{-1}\left(\delta^{-1}\right)\right)=\left(\sigma(g) g^{-1}, \theta_{1}^{-1}\left(\delta_{1}^{*-1}\right)\right) .
$$

This is an element of $H^{1}\left(F, S_{\mathrm{sc}} \stackrel{1-\theta_{1}^{-1}}{\longrightarrow} S_{1}\right)$. We have

$$
\begin{aligned}
\Delta_{\mathrm{III}}\left[{ }^{L} C(\mathfrak{e}),{ }^{L} C^{H}\left(\mathfrak{e}_{\mathfrak{z}}\right), X\right]\left(\gamma_{1}^{-1},\right. & \left.\theta^{-1}\left(\delta^{-1}\right)\right) \\
& =\left\langle\operatorname{inv}\left(\gamma_{1}^{-1}, \theta^{-1}\left(\delta^{-1}\right)\right), A_{0}\left[{ }^{L} C(\mathfrak{e}),{ }^{L} C^{H}\left(\mathfrak{e}_{\mathfrak{z}}\right), X\right]\right\rangle_{\mathrm{KS}} .
\end{aligned}
$$

Here $A_{0}\left[{ }^{L} C(\mathfrak{e}),{ }^{L} C^{H}\left(\mathfrak{e}_{\mathfrak{z}}\right), X\right]$ is the element of $H^{1}\left(W, \hat{S}_{1} \stackrel{1-\hat{\theta}_{1}^{-1}}{\longrightarrow} \hat{S}_{\mathrm{ad}}\right)$, constructed as above, but with respect to the endoscopic datum ${ }^{L} C(\mathfrak{e})$ and the $z$-pair ${ }^{L} C^{H}\left(\mathfrak{z}_{\mathfrak{e}}\right)$, rather than $\mathfrak{e}$ and $\mathfrak{z}_{\mathfrak{e}}$. Thus $\left.A_{0}\left[{ }^{L} C(\mathfrak{e}),{ }^{L} C^{H}\left(\mathfrak{e}_{\mathfrak{z}}\right), X\right]=\left(\tilde{a}_{S}[X]\right]^{-1}, s_{S}\right)$, with $\tilde{a}_{S}[X](w)=$ $\left(\tilde{t}_{1}^{-1}, \tilde{t}\right)$, and

$$
\begin{aligned}
\xi_{1}^{1}[X](\tilde{t} \times w) & ={ }^{L} \theta \circ{ }^{L} C \circ \xi(u(w)), \\
\xi_{S_{H}}^{1}[X]\left(\tilde{t}_{1} \times w\right) & ={ }^{L} C^{H} \circ \xi_{H_{1}}(u(w)) .
\end{aligned}
$$

Using (10) we see that

$$
\begin{aligned}
\xi_{1}^{1}[X](\tilde{t} \times w) & ={ }^{L} \theta \circ{ }^{L} C \circ \xi_{1}^{1}[X](t \times w), \\
\xi_{S_{H}}^{1}[X]\left(\tilde{t}_{1} \times w\right) & ={ }^{L} C^{H} \circ \xi_{S_{H}}^{1}[X]\left(t_{1} \times w\right) .
\end{aligned}
$$

According to Lemma 5.2 this is equivalent to

$$
\begin{aligned}
\xi_{1}^{1}[X](\tilde{t} \times w) & ={ }^{L} \theta \circ \xi_{1}^{1}[-X]\left(t^{-1} \times w\right), \\
\xi_{S_{H}}^{1}[X]\left(\tilde{t_{1}} \times w\right) & =\xi_{S_{H}}^{1}[-X]\left(t_{1}^{-1} \times w\right) .
\end{aligned}
$$

We conclude that

$$
\tilde{a}_{S}[X](w)=\hat{\theta}_{1}\left(a_{S}[-X](w)^{-1}\right) .
$$

The isomorphism of complexes

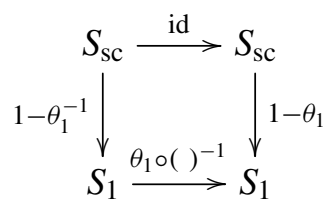


induces an isomorphism $H^{1}\left(F, S_{\mathrm{sc}} \stackrel{1-\theta_{1}^{-1}}{\longrightarrow} S_{1}\right) \rightarrow H^{1}\left(F, S_{\mathrm{sc}} \stackrel{1-\theta_{1}}{\longrightarrow} S_{1}\right)$ which, by (11), sends $\operatorname{inv}\left(\gamma_{1}^{-1}, \theta^{-1}\left(\delta^{-1}\right)\right)$ to $\operatorname{inv}\left(\gamma_{1}, \delta\right)$. The dual isomorphism of complexes

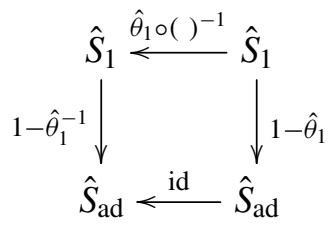

induces an isomorphism $H^{1}\left(W, \hat{S}_{1} \stackrel{1-\hat{\theta}_{1}}{\longrightarrow} \hat{S}_{\mathrm{ad}}\right) \rightarrow H^{1}\left(W, \hat{S}_{1} \stackrel{1-\hat{\theta}_{1}^{-1}}{\longrightarrow} \hat{S}_{\mathrm{ad}}\right)$ which, by (12), sends $A_{0}\left[\mathfrak{e}, \mathfrak{z}_{\mathfrak{e}},-X\right]$ to $A_{0}\left[{ }^{L} C(\mathfrak{e}),{ }^{L} C^{H}\left(\mathfrak{z}_{\mathfrak{e}}\right), X\right]$. We conclude that

$$
\Delta_{\mathrm{III}}\left[{ }^{L} C(\mathfrak{e}),{ }^{L} C^{H}\left(\mathfrak{z}_{\mathfrak{e}}\right), X\right]\left(\gamma_{1}^{-1}, \theta^{-1}\left(\delta^{-1}\right)\right)=\Delta_{\mathrm{III}}\left[\mathfrak{e}, \mathfrak{z}_{\mathfrak{e}},-X\right]\left(\gamma_{1}, \delta\right) .
$$

Combining (6), (7), (8), (9), and (13), we obtain

$$
\begin{aligned}
\Delta_{D}\left[\psi,{ }^{L} C(\mathfrak{e}),{ }^{L} C^{H}\left(\mathfrak{z}_{\mathfrak{e}}\right)\right]\left(\gamma_{1}^{-1}, \theta^{-1}\left(\delta^{-1}\right)\right) \\
=\epsilon\left(V_{G, H}, \psi_{F}\right) \cdot \Delta_{\mathrm{I}}^{\mathrm{new}}[\operatorname{spl}, A]\left(\gamma_{1}^{-1}, \theta^{-1}\left(\delta^{-1}\right)\right) \cdot \Delta_{\mathrm{II}}^{-1}[A, X]\left(\gamma_{1}^{-1}, \theta^{-1}\left(\delta^{-1}\right)\right) \\
\cdot \Delta_{\mathrm{IIII}}\left[{ }^{L} C(\mathfrak{e}),{ }^{L} C^{H}\left(\mathfrak{z}_{\mathfrak{e}}\right), X\right]\left(\gamma_{1}^{-1}, \theta^{-1}\left(\delta^{-1}\right)\right) \cdot \Delta_{\mathrm{IV}}\left(\gamma_{1}^{-1}, \theta^{-1}\left(\delta^{-1}\right)\right) \\
=\epsilon\left(V_{G, H}, \psi_{F}\right) \cdot \Delta_{\mathrm{I}}^{\text {new }}[\operatorname{spl}, A]\left(\gamma_{1}, \delta\right) \cdot \Delta_{\mathrm{II}}^{-1}[-A,-X]\left(\gamma_{1}, \delta\right) \\
\cdot \Delta_{\mathrm{III}}\left[\mathfrak{e}, \mathfrak{z}_{\mathfrak{e}},-X\right]\left(\gamma_{1}, \delta\right) \cdot \Delta_{\mathrm{IV}}\left(\gamma_{1}, \delta\right) .
\end{aligned}
$$

Since $-X$ and $-A$ are valid choices of $\chi$-data and $a$-data, according to (6) the second product is almost equal to $\Delta_{\psi}\left[\psi, \mathfrak{e}, \mathfrak{z}_{\mathfrak{e}}\right]\left(\gamma_{1}, \delta\right)$. The only difference is that the $a$-data occurring in $\Delta_{\mathrm{I}}$ is $A$, while the one occurring in $\Delta_{\mathrm{II}}$ is $-A$. Let - spl be the splitting $\left(T, B,\left\{-X_{\alpha}\right\}\right)$; then - spl and the character $\psi_{F}^{-1}$ give rise to the fixed Whittaker datum $(B, \psi)$, just like the splitting spl and the character $\psi_{F}$ did. Thus

$$
\begin{aligned}
\epsilon\left(V_{G, H}, \psi_{F}\right) \cdot \Delta_{\mathrm{I}}[\mathrm{spl}, A] & =\epsilon\left(V_{G, H}, \psi_{F}^{-1}\right) \cdot \Delta_{\mathrm{I}}[-\mathrm{spl}, A] \\
& =\epsilon\left(V_{G, H}, \psi_{F}^{-1}\right) \cdot \Delta_{\mathrm{I}}[\mathrm{spl},-A],
\end{aligned}
$$

with the first equality following from the argument of [Kottwitz and Shelstad 1999, Section 5.3], and the second from Lemma 5.1. Noting that spl and $\psi_{F}^{-1}$ give rise to the Whittaker datum $\left(B, \psi^{-1}\right)$, we obtain

$$
\Delta_{D}\left[\psi,{ }^{L} C(\mathfrak{e}),{ }^{L} C^{H}\left(\mathfrak{z}_{\mathfrak{e}}\right)\right]\left(\gamma_{1}^{-1}, \theta^{-1}\left(\delta^{-1}\right)\right)=\Delta_{D}\left[\psi^{-1}, \mathfrak{e}, \mathfrak{z}_{\mathfrak{e}}\right]\left(\gamma_{1}, \delta\right) .
$$

Corollary 5.5. Let $f \in \mathscr{H}(G)$ and $f^{H_{1}} \in \mathscr{H}\left(H_{1}\right)$ be functions such that the $\left(\theta^{-1}, \omega\right)$ twisted orbital integrals of $f$ match the stable orbital integrals of $f^{H_{1}}$ with respect to $\tilde{\Delta}\left[\psi^{-1},{ }^{L} C(e),{ }^{L} C^{H}\left(z_{e}\right)\right]$. Then the $(\theta, \omega)$-twisted orbital integrals of $f \circ \theta^{-1} \circ i$ match the stable orbital integrals of $f^{H_{1}} \circ i$ with respect to $\tilde{\Delta}\left[\psi, e, z_{e}\right]$. Here $\tilde{\Delta}$ stands for any of the two (respectively, four) Whittaker normalizations of the transfer 
factor for twisted (respectively, standard) endoscopy, and $i$ is the map on $G(F)$ or $H_{1}(F)$ sending every element to its inverse.

Proof. $\mathrm{SO}\left(\gamma_{1}, f^{H_{1}} \circ i\right)$

$$
\begin{aligned}
= & \operatorname{SO}\left(\gamma_{1}^{-1}, f^{H_{1}}\right) \\
= & \sum_{\delta \in G(F) / \theta^{-1} \_\sim} \tilde{\Delta}\left[\psi^{-1},{ }^{L} C(e),{ }^{L} C^{H}\left(z_{e}\right)\right]\left(\gamma_{1}^{-1}, \delta\right) O^{\theta^{-1}, \omega}(\delta, f) \\
= & \sum_{\delta \in G(F) / \theta^{-1} \_\sim} \tilde{\Delta}\left[\psi^{-1},{ }^{L} C(e),{ }^{L} C^{H}\left(z_{e}\right)\right]\left(\gamma_{1}^{-1}, \delta\right) O^{\theta, \omega}\left(\theta\left(\delta^{-1}\right), f \circ \theta^{-1} \circ i\right) \\
= & \sum_{\delta^{\prime} \in G(F) / \theta-\sim} \tilde{\Delta}\left[\psi, e, z_{e}\right]\left(\gamma_{1}, \delta^{\prime}\right) O^{\theta, \omega}\left(\delta^{\prime}, f \circ \theta^{-1} \circ i\right) .
\end{aligned}
$$

The last line follows from Proposition 5.4, with the substitution $\delta^{\prime}=\theta\left(\delta^{-1}\right)$.

Fact 5.6. Assume that $\theta$ has finite order. Let $\pi$ be an irreducible admissible tempered $(B, \psi)$-generic $\theta$-stable representation of $G(F)$, and let $A: \pi \rightarrow \pi \circ \theta$ be the unique isomorphism which preserves a $(B, \psi)$-Whittaker functional. Then the dual map $A^{\vee}:(\pi \circ \theta)^{\vee} \rightarrow \pi^{\vee}$ preserves a $\left(B, \psi^{-1}\right)$-Whittaker functional.

Proof. Let $V$ be the vector space on which $\pi$ acts. Since $\pi$ is tempered, it is unitary. Let $\langle\cdot, \cdot\rangle$ be a $\pi$-invariant nondegenerate Hermitian form on $V$. Then

$$
\bar{V} \rightarrow V^{\vee}, \quad w \mapsto\langle\cdot, w\rangle
$$

is a $\pi-\pi^{\vee}$-equivariant isomorphism, and it identifies $A^{\vee}$ with the $\langle\cdot, \cdot\rangle$-adjoint of $A$, which we will call $A^{*}$. We claim that $A^{*}=A^{-1}$. Indeed, $(v, w) \mapsto\langle A v, A w\rangle$ is another $\pi$-invariant scalar product, hence there exists a scalar $c \in \mathbb{C}^{\times}$with $\langle A v, A w\rangle=c\langle v, w\rangle$. On the one hand, since both sides are Hermitian, this scalar must belong to $\mathbb{R}_{>0}$. On the other hand, since $\theta$ has finite order, so does $A$, and thus $c$ must be a root of unity. This shows that $c=1$, hence $A^{*}=A^{-1}$. Let $\sigma$ denote complex conjugation. If $\lambda: V \rightarrow \mathbb{C}$ is a $(B, \psi)$-Whittaker functional preserved by $A$, then $\sigma \circ \lambda: \bar{V} \rightarrow \mathbb{C}$ is a $\left(B, \psi^{-1}\right)$-Whittaker functional preserved by $A^{\vee}=A^{*}=A^{-1}$.

Corollary 5.7. If $\tilde{\pi}$ is the unique extension of $\pi$ to a representation of $G(F) \rtimes\langle\theta\rangle$ so that $\tilde{\pi}(\theta)$ is the isomorphism $\pi \rightarrow \pi \circ \theta$ which fixes $a(B, \psi)$-Whittaker functional, then $\tilde{\pi}^{\vee}$ is the unique extension of $\pi^{\vee}$ to a representation of $G(F) \rtimes\langle\theta\rangle$ so that $\tilde{\pi}^{\vee}(\theta)$ is the isomorphism $\pi^{\vee} \rightarrow \pi^{\vee} \circ \theta$ which fixes a $\left(B, \psi^{-1}\right)$-Whittaker functional.

Let us recall Theorem 7.1(a) of [Adams and Vogan 2012]. For any Langlands parameter $\varphi: W \rightarrow{ }^{L} G$ for a real connected reductive group $G$ with corresponding $L$-packet $\Pi_{\varphi}$, the theorem shows that the set $\left\{\pi^{\vee} \mid \pi \in \Pi_{\varphi}\right\}$ is also an $L$-packet, and its parameter is ${ }^{L} C \circ \varphi$. Assume that $\varphi$ is tempered, and denote by $S \Theta_{\varphi}$ 
the stable character of the $L$-packet $\Pi_{\varphi}$. Then an immediate corollary is that $S \Theta_{\varphi} \circ i=S \Theta_{L_{C} \circ \varphi}$. We will now prove this equality for quasisplit symplectic and special orthogonal $p$-adic groups. After that, we will use it to derive formula (2). With this formula at hand, we will derive the precise $p$-adic analog of Adams and Vogan's Theorem 7.1(a) as a corollary.

Theorem 5.8. Let $H$ be a quasisplit symplectic or special orthogonal group and $\varphi: W^{\prime} \rightarrow{ }^{L} H$ a tempered Langlands parameter. Write $S \Theta_{\varphi}$ for the stable character of the L-packet attached to $\varphi$. Then we have an equality of linear forms on $\tilde{\mathscr{H}}(H)$ :

$$
S \Theta_{\varphi} \circ i=S \Theta_{{ }^{L} C^{H} \circ \varphi} .
$$

Proof. We recall very briefly the characterizing property of $S \Theta_{\varphi}$, following Arthur [2013, Section 1 and Section 2]. Let $G=\mathrm{GL}_{n} / F$ and let spl $=\left(T, B,\left\{X_{\alpha}\right\}\right)$ be the standard splitting consisting of the subgroup $T$ of diagonal matrices, the subgroup $B$ of upper triangular matrices, and the set $\left\{X_{\alpha}\right\}$ of elementary matrices whose entries are zero except for one entry in the first superdiagonal, which is equal to 1 . Let $\theta$ be the outer automorphism of $G$ preserving spl. Equip $\hat{G}=\mathrm{GL}_{n}(\mathbb{C})$ with its standard splitting $\left(\hat{T}, \hat{B},\left\{\hat{X}_{\alpha}\right\}\right)$ and let $\hat{\theta}$ be the outer automorphism of $\hat{G}$ preserving that splitting. The standard representation $\hat{H} \rightarrow \hat{G}$ can be extended to an $L$-embedding $\xi:{ }^{L} H \rightarrow{ }^{L} G$ and augmented by an element $s \in \hat{T}$ to provide an endoscopic datum $\mathfrak{e}=\left(H,{ }^{L} H, s, \xi\right)$ for the triple $(G, \theta, 1)$. Then $\xi \circ \varphi$ is a Langlands parameter for $G$ invariant under $\hat{\theta}$. Let $\pi$ be the representation of $G(F)$ assigned to $\xi \circ \varphi$ by the local Langlands correspondence [Harris and Taylor 2001; Henniart 2000]. We have $\pi \cong \pi \circ \theta$. Choosing an additive character $\psi_{F}: F \rightarrow \mathbb{C}^{\times}$ we obtain from the standard splitting of $G$ a $\theta$-stable Whittaker datum $(B, \psi)$. There is a unique isomorphism $A: \pi \rightarrow \pi \circ \theta$ which preserves one (hence all) $(B, \psi)$-Whittaker functionals. Then we have the distribution

$$
f \mapsto T \Theta_{\xi \circ \varphi}^{\psi}(f)=\operatorname{tr}\left(v \mapsto \int_{G(F)} f(g) \pi(g) A v d g\right) .
$$

By construction, $S \Theta_{\varphi}$ is the unique stable distribution on $\tilde{\mathscr{H}}(H)$ with the property that

$$
S \Theta_{\varphi}\left(f^{H}\right)=T \Theta_{\xi \circ \varphi}^{\psi}(f)
$$

for all $f \in \tilde{\mathscr{H}}(G)$ and $f^{H} \in \tilde{\mathscr{H}}(H)$ such that the $(\theta, 1)$-twisted orbital integrals of $f$ match the stable orbital integrals of $f^{H}$ with respect to $\Delta^{\prime}\left[\psi, \mathfrak{e}, \mathfrak{z}_{\mathfrak{e}}\right]$. Here $\mathfrak{z}_{\mathfrak{e}}$ stands for the tautological pair ( $H$, id).

Now consider the transfer factor $\Delta^{\prime}\left[\psi^{-1},{ }^{L} C(\mathfrak{e}),{ }^{L} C^{H}\left(\mathfrak{z}_{\mathfrak{e}}\right)\right]$. We have chosen both $\hat{C}$ and $\hat{C}^{H}$ to preserve the standard tori in $\hat{G}$ and $\hat{H}$ and act as inversion on those. Moreover the endoscopic element $s$ belongs to $\hat{T}$. Using the datum ${ }^{L} C(\mathfrak{e})$ and the pair ${ }^{L} C^{H}\left(\mathfrak{z}_{\mathfrak{e}}\right)$ has the same effect as using the datum $\left(H, \mathscr{H}, s,{ }^{L} C \circ{ }^{L} \theta \circ \xi \circ{ }^{L} C^{H^{-1}}\right)$ 
and the pair $\mathfrak{z}_{\mathfrak{e}}$. We have ${ }^{L} \theta \circ \xi=\operatorname{Int}\left(s^{-1}\right) \xi$, so replacing ${ }^{L} \theta \circ \xi$ by $\xi$ changes the above datum to an equivalent one. An easy computation shows furthermore that ${ }^{L} C \circ \xi \circ{ }^{L} C^{H^{-1}}=\operatorname{Int}(t) \circ \xi$ for a suitable $t \in \hat{T}$. All in all, up to equivalence, we see that replacing $\mathfrak{e}$ and $\mathfrak{z}_{\mathfrak{e}}$ by ${ }^{L} C(\mathfrak{e})$ and ${ }^{L} C^{H}\left(\mathfrak{z}_{\mathfrak{e}}\right)$ has no effect, and this implies that

$$
\Delta^{\prime}\left[\psi^{-1},{ }^{L} C(\mathfrak{e}),{ }^{L} C^{H}\left(\mathfrak{z}_{\mathfrak{e}}\right)\right]=\Delta^{\prime}\left[\psi^{-1}, \mathfrak{e}, \mathfrak{z}_{\mathfrak{e}}\right] .
$$

Let us abbreviate this factor to $\Delta^{\prime}\left[\psi^{-1}\right]$.

We have $S \Theta_{L_{C} C^{H} \varphi}\left(f^{H}\right)=T \Theta_{\xi_{\circ}{ }^{L} C^{H} \circ \varphi}^{\psi}(f)$. As we just argued, $\xi \circ{ }^{L} C^{H}$ is $\hat{G}$ conjugate to ${ }^{L} C \circ \xi$. Thus, the Galois representation $\xi \circ{ }^{L} C^{H} \circ \varphi$ is the contragredient to the Galois representation $\xi \circ \varphi$. As the local Langlands correspondence for $\mathrm{GL}_{n}$ respects the operation of taking the contragredient, Corollary 5.7 implies that

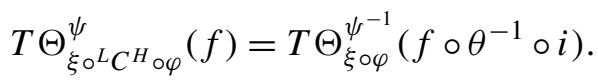

By construction of $S \Theta_{\xi \circ \varphi}$, we have

$$
T \Theta_{\xi \circ \varphi}^{\psi^{-1}}\left(f \circ \theta^{-1} \circ i\right)=S \Theta_{\xi \circ \varphi}\left(f^{H}\right)
$$

whenever' $f^{H}$ is an element of $\tilde{\mathscr{H}}(H)$ whose stable orbital integrals match the $(\theta, 1)$ orbital integrals of $f \circ \theta^{-1} \circ i$ with respect to $\Delta^{\prime}\left[\psi^{-1}\right]$. By Corollary 5.5, $f^{H} \circ i$ is such a function, and we see that the distribution $f \mapsto S \Theta_{\xi \circ \varphi}\left(f^{H} \circ i\right)$ satisfies the property that characterizes $S \Theta_{{ }^{L} C_{H} \circ \varphi}$, hence must be equal to the latter.

Theorem 5.9. Let $G$ be a quasisplit real $K$-group or a quasisplit symplectic or special orthogonal $p$-adic group, and let $(B, \psi)$ be a Whittaker datum. Let $\varphi$ : $W^{\prime} \rightarrow{ }^{L} G$ be a tempered Langlands parameter, and $\rho \in \operatorname{Irr}\left(\mathscr{Y}_{\varphi}\right)$. Then

$$
\iota_{B, \psi}(\varphi, \rho)^{\vee}=\iota_{B, \psi^{-1}}\left({ }^{L} C \circ \varphi,\left[\rho \circ \hat{C}^{-1}\right]^{\vee}\right) .
$$

Proof. Put $\pi=\iota_{B, \psi}(\varphi, \rho)$. For each semisimple $s \in S_{\varphi}$, let $\mathfrak{e}_{s}=(H, \mathcal{H}, s, \xi)$ be the corresponding endoscopic datum (see Section 3), and choose a $z$-pair $\mathfrak{z}_{s}=\left(H_{1}, \xi_{H_{1}}\right)$. We have the Whittaker normalization $\Delta\left[\psi, \mathfrak{e}_{s}, \mathfrak{z}_{s}\right]$ of the transfer factor compatible with [Langlands and Shelstad 1987] (see the discussion before Proposition 5.4).

By construction, $\varphi$ factors through $\xi$. Put $\varphi_{s}=\varphi \circ \xi_{H_{1}}$. For any function $f \in \tilde{\mathscr{H}}(G)$ let $f^{s, \psi} \in \tilde{\mathscr{H}}\left(H_{1}\right)$ be such that $f$ and $f^{s, \psi}$ have $\Delta\left[\psi, \mathfrak{e}_{s}, \mathfrak{z}_{s}\right]$-matching orbital integrals. Then the distribution

$$
f \mapsto S \Theta_{\varphi_{s}}\left(f^{s, \psi}\right)
$$

is independent of the choices of $f^{s, \psi}$ and $\mathfrak{z}_{s}$. As discussed in Section 3, we have the inversion of endoscopic transfer

$$
\Theta_{\pi}(f)=\left|\mathscr{Y}_{\varphi}\right|^{-1} \sum_{s \in \mathscr{S}_{\varphi}} \overline{\langle s, \rho\rangle} S \Theta_{\varphi_{s}}\left(f^{s, \psi}\right) .
$$


Thus, we need to show that

$$
\Theta_{\pi^{\vee}}(f)=\left|\mathscr{S}_{L_{C} \circ \varphi}\right|^{-1} \sum_{s^{\prime} \in \mathscr{I}_{L_{C} \varphi}} \overline{\left\langle\hat{C}^{-1}\left(s^{\prime}\right), \rho^{\vee}\right\rangle} S \Theta_{\left[{ }^{L} C \circ \varphi\right]_{s^{\prime}}}\left(f^{s^{\prime}, \psi^{-1}}\right) .
$$

Reindexing the sum using $s^{\prime}=\hat{C}\left(s^{-1}\right)$, we can write the right-hand side as

$$
\left|\mathscr{S}_{\varphi}\right|^{-1} \sum_{s \in \mathscr{S}_{\varphi}} \overline{\langle s, \rho\rangle} S \Theta_{\left[{ }^{L} C \circ \varphi\right]_{s^{\prime}}}\left(f^{s^{\prime}, \psi^{-1}}\right) .
$$

The theorem will be proved once we show

$$
S \Theta_{\varphi_{s}}\left([f \circ i]^{s, \psi}\right)=S \Theta_{\left[{ }^{L} C \circ \varphi\right]_{s^{\prime}}}\left(f^{s^{\prime}, \psi^{-1}}\right) .
$$

The endoscopic datum corresponding to ${ }^{L} C \circ \varphi$ and $s^{\prime}$ is precisely ${ }^{L} C\left(\mathfrak{e}_{s}\right)$ (in the sense that $1 \in \mathrm{GL}_{n}(\mathbb{C})$ is an isomorphism between the two). We are free to choose any $z$-pair for it, and we choose ${ }^{L} C^{H}\left(\mathfrak{z}_{s}\right)$. Then $\left[{ }^{L} C \circ \varphi\right]_{s^{\prime}}={ }^{L} C^{H_{1}} \circ \varphi_{s}$ and Theorem 5.8 in the $p$-adic case and [Adams and Vogan 2012, Theorem 7.1(a)] in the real case imply

$$
S \Theta_{\left[{ }^{L} C \circ \varphi\right]_{s^{\prime}}}\left(f^{s^{\prime}, \psi^{-1}}\right)=S \Theta_{\varphi_{s}}\left(f^{\left.s^{\prime}, \psi^{-1} \circ i\right) .}\right.
$$

The functions $f$ and $f^{s^{\prime}, \psi^{-1}}$ have $\Delta\left[\psi^{-1},{ }^{L} C\left(\mathfrak{e}_{s}\right),{ }^{L} C^{H}\left(\mathfrak{z}_{s}\right)\right]$-matching orbital integrals. By Corollary 5.5, the functions $f \circ i$ and $f^{s^{\prime}, \psi^{-1}} \circ i$ have $\Delta\left[\psi, \mathfrak{e}, \mathfrak{z}_{\mathfrak{e}}\right]$-matching orbital integrals. It follows that the functions $[f \circ i]^{s, \psi}$ and $f^{s^{\prime}, \psi^{-1}} \circ i$ have the same stable orbital integrals, and the theorem follows.

We alert the reader that, as was explained in Section 3, the symbol $\iota_{B, \psi}(\varphi, \rho)$ refers to an individual representation of $G(F)$ in all cases of Theorem 5.9, except possibly when $G$ is an even orthogonal $p$-adic group, in which case Arthur's classification may assign to the pair $(\varphi, \rho)$ a pair of representations, rather than an individual representation. In that case, the theorem asserts that if $\left\{\pi_{1}, \pi_{2}\right\}$ is the pair of representations associated with $(\varphi, \rho)$, then $\left\{\pi_{1}^{\vee}, \pi_{2}^{\vee}\right\}$ is the pair of representations associated with $\iota_{B, \psi^{-1}}\left({ }^{L} C \circ \varphi,\left[\rho \circ \hat{C}^{-1}\right]^{\vee}\right)$.

The following is the $p$-adic analog of [Adams and Vogan 2012, Theorem 7.1(a)].

Corollary 5.10. Let $G$ be a quasisplit symplectic or special orthogonal p-adic group, and let $\varphi: W^{\prime} \rightarrow{ }^{L} G$ be a tempered Langlands parameter. If $\Pi$ is an $L$-packet assigned to $\varphi$, then

$$
\Pi^{\vee}=\left\{\pi^{\vee} \mid \pi \in \Pi\right\}
$$

is an L-packet assigned to ${ }^{L} C \circ \varphi$.

Proof. When $G$ is either a symplectic or an odd orthogonal group, the statement follows immediately from Theorem 5.9. However, if $G$ is an even orthogonal group, $\Pi$ is one of two $L$-packets $\Pi_{1}, \Pi_{2}$ assigned to $\varphi$, and a priori we only know that 
the set $\Pi^{\vee}$ belongs to the union of the two $L$-packets $\Pi_{1}^{\prime}, \Pi_{2}^{\prime}$ assigned to ${ }^{L} C \circ \varphi$. We claim that in fact it equals one of these two $L$-packets. Indeed, let $S \Theta$ be the stable character of $\Pi$. This is now a stable linear form on $\mathscr{H}(G)$, not just on $\tilde{\mathscr{H}}(G)$. The linear form $S \Theta \circ i$ is still stable. If $S \Theta_{1}^{\prime}$ and $S \Theta_{2}^{\prime}$ are the stable characters of $\Pi_{1}^{\prime}$ and $\Pi_{2}^{\prime}$ respectively, then the restrictions of $S \Theta_{1}^{\prime}$ and $S \Theta_{2}^{\prime}$ to $\tilde{\mathscr{H}}(G)$ are equal, and moreover according to Theorem 5.8 these restrictions are equal to the restriction of $S \Theta \circ i$ to $\tilde{\mathscr{H}}(G)$. From [Arthur 2013, Corollary 8.4.5] we conclude that

$$
S \Theta \circ i=\lambda S \Theta_{1}^{\prime}+\mu S \Theta_{2}^{\prime}
$$

for some $\lambda, \mu \in \mathbb{C}$ with $\lambda+\mu=1$. However, each of the three distributions $S \Theta \circ i, S \Theta_{1}^{\prime}, S \Theta_{2}^{\prime}$ is itself a sum of characters of tempered representations. Since $\Pi_{1}^{\prime}$ and $\Pi_{2}^{\prime}$ are disjoint, the linear independence of these characters then forces one of the numbers $\lambda, \mu$ to be equal to 1 , and the other to 0 .

\section{Depth-zero and epipelagic $L$-packets of $p$-adic groups}

In this section, we are going to examine two constructions of $L$-packets on general reductive $p$-adic groups and show that (2) is satisfied by these $L$-packets.

The first construction is that of [DeBacker and Reeder 2009], in which $L$-packets consisting of depth-zero supercuspidal representations are constructed for each pure inner form of an unramified $p$-adic group. This construction was then extended to inner forms of $p$-adic groups arising from isocrystals with additional structure [Kaletha 2011]. The second construction is that of [Kaletha 2012], in which $L$ packets consisting of epipelagic representations are constructed for each tamely ramified $p$-adic group. The notion of epipelagic representation was introduced and studied by Reeder and Yu in [2012].

Fix a Langlands parameter $\varphi: W \rightarrow{ }^{L} G$ of the type considered in [DeBacker and Reeder 2009] or [Kaletha 2012]. Fix a $\Gamma$-invariant splitting $\left(\hat{T}, \hat{B},\left\{X_{\hat{\alpha}}\right\}\right)$ of $\hat{G}$ and arrange that $\hat{T}$ is the unique torus normalized by $\varphi$. Choose a Chevalley involution $\hat{C}$ which sends the fixed splitting to its opposite. Then $\hat{C}$ commutes with all automorphisms preserving the fixed splitting, in particular with the action of $\Gamma$ on $\hat{G}$, and hence ${ }^{L} C=\hat{C} \times \mathrm{id}_{W}$ is an $L$-automorphism. Moreover, the action of $\hat{C}$ on $N(\hat{T}, \hat{G})$ preserves $\hat{T}$ and thus induces an action on $\Omega(\hat{T}, \hat{G})$. Since $\hat{C}\left(X_{\hat{\alpha}}\right)=X_{-\hat{\alpha}}$, this action fixes each simple reflection and is therefore trivial.

In both constructions of $L$-packets the first step is to form the $\Gamma$-module $\hat{S}$ with underlying abelian group $\hat{T}$ and $\Gamma$-action given by the composition $\Gamma \rightarrow$ $\Omega(\hat{T}, \hat{G}) \rtimes \Gamma$ of $\varphi$ and the natural projection $N(\hat{T}, \hat{G}) \rightarrow \Omega(\hat{T}, \hat{G})$. By the argument of the preceding paragraph, the $\Gamma$-module $\hat{S}$ for ${ }^{L} C \circ \varphi$ is the same as the one for $\varphi$.

The next step is to obtain from $\varphi$ a character $\chi: S(F) \rightarrow \mathbb{C}^{\times}$. This is done by factoring $\varphi={ }^{L} j_{X} \circ \varphi_{S}$, where ${ }^{L} j_{X}:{ }^{L} S \rightarrow{ }^{L} G$ is an $L$-embedding constructed 
from $\varphi$, and $\varphi_{S}: W \rightarrow{ }^{L} S$ is a Langlands parameter for $S$, and then letting $\chi$ be the character corresponding to $\varphi_{S}$. For the depth-zero case, this is the reinterpretation given in [Kaletha 2011], and the $L$-embedding ${ }^{L} S \rightarrow{ }^{L} G$ is obtained by choosing arbitrary unramified $\chi$-data $X$ for $R(\hat{S}, \hat{G})$. Applying Lemma 5.2 to the equation $\varphi={ }^{L} j_{X} \circ \varphi_{S}$ we see that

$$
{ }^{L} C \circ \varphi={ }^{L} j_{-X} \circ(-1) \circ \varphi_{S} .
$$

Since $-X$ is another set of unramified $\chi$-data, and it is shown in [Kaletha 2011, Section 3.4] that $\varphi_{S}$ is independent of the choice of $X$, we see that $\left[{ }^{L} C \circ \varphi\right]_{S}=$ $(-1) \circ \varphi_{S}$. In other words, the character of $S(F)$ constructed from ${ }^{L} C \circ \varphi$ is $\chi_{S}^{-1}$.

We claim that the same is true in the epipelagic case. That case is a bit more subtle because ${ }^{L} j_{X}$ depends on $\varphi$ more strongly - the $\chi$-data $X$ is chosen based on the restriction of $\varphi$ to wild inertia. What we need to show is that if $X$ is chosen for $\varphi$, then the choice for ${ }^{L} C \circ \varphi$ is $-X$. This however follows right away from the fact that the restriction of ${ }^{L} C \circ \varphi$ to wild inertia equals the composition of $(-1)$ with the restriction of $\varphi$ to wild inertia.

The third step in the construction of both kinds of $L$-packets relies on a procedure (different in the two cases) which associates to an admissible embedding $j$ of $S$ into an inner form $G^{\prime}$ of $G$ a representation $\pi\left(\chi_{S}, j\right)$ of $G^{\prime}(F)$. We won't recall this procedure - for our current purposes it will be enough to treat it as a black box. The only feature of this black box that is essential for us is that the contragredient of $\pi\left(\chi_{S}, j\right)$ is given by $\pi\left(\chi_{S}^{-1}, j\right)$. Now let $(B, \psi)$ be a Whittaker datum for $G$. It is shown in both cases that there exists an admissible embedding $j_{0}: S \rightarrow G$, unique up to $G(F)$-conjugacy, so that the representation $\pi\left(\chi_{S}, j_{0}\right)$ of $G(F)$ is $(B, \psi)$-generic. Moreover, one has $S_{\varphi}=[\hat{S}]^{\Gamma}$, so that $\operatorname{Irr}\left(S_{\varphi}\right)=X^{*}\left(\hat{S}^{\Gamma}\right)=X_{*}(S)_{\Gamma}=\boldsymbol{B}(S)$, where $\boldsymbol{B}(S)$ is the set of isomorphism classes of isocrystals with $S$-structure [Kottwitz 1997]. Using $j_{0}$ one obtains a map $\operatorname{Irr}\left(S_{\varphi}\right)=\boldsymbol{B}(S) \rightarrow \boldsymbol{B}(G)_{\text {bas. }}$. Each $\rho \in \operatorname{Irr}\left(S_{\varphi}\right)$ provides in this way an extended pure inner twist $\left(G^{b_{\rho}}, b_{\rho}, \xi_{\rho}\right)$. The composition $j_{\rho}=\xi_{\rho} \circ j_{0}$ is an admissible embedding $S \rightarrow G^{b_{\rho}}$ defined over $F$ and provides by the black box construction alluded to above a representation $\pi\left(\chi_{S}, j_{\rho}\right)$ of $G^{b_{\rho}}(F)$. The construction of $L$-packets and their internal parametrization is then realized by

$$
\iota_{B, \psi}: \operatorname{Irr}\left(S_{\varphi}\right) \rightarrow \Pi_{\varphi}, \quad \rho \mapsto \pi\left(\chi_{S}, j_{\rho}\right) .
$$

The contragredient of $\pi\left(\chi_{S}, j_{0}\right)$ is given by $\pi\left(\chi_{S}^{-1}, j_{0}\right)$, and the latter representation is $\left(B, \psi^{-1}\right)$-generic. Hence, the version of $j_{0}$ associated to ${ }^{L} C \circ \varphi$ and the Whittaker datum $\left(B, \psi^{-1}\right)$ is equal to $j_{0}$. Using $S_{L_{C} \circ \varphi}=\left[\hat{S}^{\Gamma}\right]$ and $\rho^{\vee} \circ \hat{C}^{-1}=\rho$, and reviewing the procedure above, we see that

$$
\iota_{B, \psi^{-1}}\left({ }^{L} C \circ \varphi, \rho^{\vee} \circ \hat{C}^{-1}\right)=\pi\left(\chi_{S}^{-1}, j_{\rho}\right)=\pi\left(\chi_{S}, j_{\rho}\right)^{\vee}=\iota_{B, \psi}(\varphi, \rho)^{\vee} .
$$




\section{Acknowledgements}

The author would like to thank Jeffrey Adams for discussing with him the paper [Adams and Vogan 2012], Diana Shelstad for enlightening discussions concerning [Kottwitz and Shelstad 2012] and the normalizations of transfer factors, Robert Kottwitz and Peter Sarnak for their reading of an earlier draft of this paper, Sandeep Varma for pointing out an inaccuracy in an earlier draft, and Dipendra Prasad for pointing out that equations very similar to (1) and (2) appear as conjectures in [Gan, Gross, and Prasad 2012] and [Prasad 2012].

\section{References}

[Adams and Vogan 2012] J. D. Adams and D. A. Vogan, “The contraingredient”, preprint, 2012. arXiv 1201.0496

[Adams et al. 1992] J. Adams, D. Barbasch, and D. A. Vogan, Jr., The Langlands classification and irreducible characters for real reductive groups, Progress in Mathematics 104, Birkhäuser, Boston, 1992. MR 93j:22001 Zbl 0756.22004

[Arthur 1999] J. Arthur, “On the transfer of distributions: weighted orbital integrals", Duke Math. J. 99:2 (1999), 209-283. MR 2000i:22023 Zbl 0938.22019

[Arthur 2013] J. Arthur, The endoscopic classiffication of representations: orthogonal and symplectic groups, Colloquium Publ. 61, Amer. Math. Soc., Providence, RI, 2013.

[Borel 1979] A. Borel, “Automorphic L-functions”, pp. 27-61 in Automorphic forms, representations and L-functions (Corvallis, OR, 1977), edited by A. Borel and W. Casselman, Proc. Sympos. Pure Math. 33, Amer. Math. Soc., Providence, RI, 1979. MR 81m:10056 Zbl 0412.10017

[Bourbaki 2002] N. Bourbaki, Lie groups and Lie algebras: Chapters 4-6, Springer, Berlin, 2002. MR 2003a:17001 Zbl 0983.17001

[DeBacker and Reeder 2009] S. DeBacker and M. Reeder, "Depth-zero supercuspidal $L$-packets and their stability", Ann. of Math. (2) 169:3 (2009), 795-901. MR 2010d:22023 Zbl 1193.11111

[Gan, Gross, and Prasad 2012] W. T. Gan, B. H. Gross, and D. Prasad, "Symplectic local root numbers, central critical $L$-values, and restriction problems in the representation theory of classical groups”, pp. 1-109 in Sur les conjectures de Gross and Prasad, vol. I, Astérisque 346, Société Mathématique de France, Paris, 2012. Zbl 06154194

[Harris and Taylor 2001] M. Harris and R. Taylor, The geometry and cohomology of some simple Shimura varieties, Annals of Mathematics Studies 151, Princeton University Press, 2001. MR 2002m:11050 Zbl 1036.11027

[Henniart 2000] G. Henniart, "Une preuve simple des conjectures de Langlands pour GL(n) sur un corps $p$-adique”, Invent. Math. 139:2 (2000), 439-455. MR 2001e:11052 Zbl 1048.11092

[Kaletha 2011] T. Kaletha, "Supercuspidal $L$-packets via isocrystals", preprint, 2011. To appear in Amer. J. Math. arXiv 1004.2230

[Kaletha 2012] T. Kaletha, "Epipelagic $L$-packets and rectifying characters", preprint, 2012. arXiv 1209.1720

[Kottwitz 1984] R. E. Kottwitz, "Stable trace formula: cuspidal tempered terms", Duke Math. J. 51:3 (1984), 611-650. MR 85m:11080 Zbl 0576.22020

[Kottwitz 1997] R. E. Kottwitz, "Isocrystals with additional structure, II", Compositio Math. 109:3 (1997), 255-339. MR 99e:20061 Zbl 0966.20022 
[Kottwitz and Shelstad 1999] R. E. Kottwitz and D. Shelstad, Foundations of twisted endoscopy, Astérisque 255, Société Mathématique de France, Paris, 1999. MR 2000k:22024 Zbl 0958.22013

[Kottwitz and Shelstad 2012] R. Kottwitz and D. Shelstad, "On splitting invariants and sign conventions in endoscopic transfer”, preprint, 2012. arXiv 1201.5658

[Langlands 1989] R. P. Langlands, "On the classification of irreducible representations of real algebraic groups", pp. 101-170 in Representation theory and harmonic analysis on semisimple Lie groups, edited by P. J. Sally, Jr., Math. Surveys Monogr. 31, Amer. Math. Soc., Providence, RI, 1989. MR 91e:22017 Zbl 0741.22009

[Langlands and Shelstad 1987] R. P. Langlands and D. Shelstad, "On the definition of transfer factors”, Math. Ann. 278:1-4 (1987), 219-271. MR 89c:11172 Zbl 0644.22005

[Prasad 2012] D. Prasad, "A "relative" local Langlands correspondence", preprint, 2012, available at http://www.math.tifr.res.in/ dprasad/relative-L.pdf.

[Reeder 2008] M. Reeder, "Supercuspidal $L$-packets of positive depth and twisted Coxeter elements", J. Reine Angew. Math. 620 (2008), 1-33. MR 2009e:22019 Zbl 1153.22021

[Reeder and Yu 2012] M. Reeder and J.-K. Yu, "Epipelagic representations and invariant theory", preprint, 2012, available at https://www2.bc.edu/mark-reeder/Epipelagic.pdf. To appear in J. Amer. Math. Soc.

[Shahidi 1990] F. Shahidi, “A proof of Langlands' conjecture on Plancherel measures; complementary series for p-adic groups", Ann. of Math. (2) 132:2 (1990), 273-330. MR 91m:11095 Zbl 0780.22005

[Shelstad 2008] D. Shelstad, “Tempered endoscopy for real groups, III: Inversion of transfer and L-packet structure", Represent. Theory 12 (2008), 369-402. MR 2010c:22016 Zbl 1159.22007

[Springer 1981] T. A. Springer, Linear algebraic groups, Progress in Mathematics 9, Birkhäuser, Boston, 1981. MR 84i:20002 Zbl 0453.14022

[Tate 1979] J. Tate, "Number theoretic background", pp. 3-26 in Automorphic forms, representations and L-functions (Corvallis, OR, 1977), edited by A. Borel and W. Casselman, Proc. Sympos. Pure Math. 33, Amer. Math. Soc., Providence, RI, 1979. MR 80m:12009 Zbl 0422.12007

[Vogan 1993] D. A. Vogan, Jr., "The local Langlands conjecture", pp. 305-379 in Representation theory of groups and algebras, edited by J. Adams et al., Contemp. Math. 145, Amer. Math. Soc., Providence, RI, 1993. MR 94e:22031 Zbl 0802.22005

Communicated by Richard Taylor

Received 2012-07-14 Revised 2013-01-25 Accepted 2013-04-26

tkaletha@math.princeton.edu Department of Mathematics, Princeton University, Fine Hall, Washington Road, Princeton, NJ 08544, United States 


\section{Algebra \& Number Theory}

msp.org/ant

\section{EDITORS}

MANAGING EDITOR

Bjorn Poonen

Massachusetts Institute of Technology

Cambridge, USA

\author{
EDITORIAL BOARD CHAIR \\ David Eisenbud \\ University of California \\ Berkeley, USA
}

\section{BOARD OF EDITORS}

Georgia Benkart

Dave Benson

Richard E. Borcherds

John H. Coates

J-L. Colliot-Thélène

Brian D. Conrad

Hélène Esnault

Hubert Flenner

Edward Frenkel

Andrew Granville

Joseph Gubeladze

Roger Heath-Brown

Ehud Hrushovski

Craig Huneke

Mikhail Kapranov

Yujiro Kawamata

János Kollár

Yuri Manin

Barry Mazur

Philippe Michel
University of Wisconsin, Madison, USA

University of Aberdeen, Scotland

University of California, Berkeley, USA

University of Cambridge, UK

CNRS, Université Paris-Sud, France

University of Michigan, USA

Freie Universität Berlin, Germany

Ruhr-Universität, Germany

University of California, Berkeley, USA

Université de Montréal, Canada

San Francisco State University, USA

Oxford University, UK

Hebrew University, Israel

University of Virginia, USA

Yale University, USA

University of Tokyo, Japan

Princeton University, USA

Northwestern University, USA

Harvard University, USA

École Polytechnique Fédérale de Lausanne
Susan Montgomery

Shigefumi Mori

Raman Parimala

Jonathan Pila

Victor Reiner

Karl Rubin

Peter Sarnak

Joseph H. Silverman

Michael Singer

Vasudevan Srinivas

J. Toby Stafford

Bernd Sturmfels

Richard Taylor

Ravi Vakil

Michel van den Bergh

Marie-France Vignéras

Kei-Ichi Watanabe

Efim Zelmanov

Shou-Wu Zhang
University of Southern California, USA

RIMS, Kyoto University, Japan

Emory University, USA

University of Oxford, UK

University of Minnesota, USA

University of California, Irvine, USA

Princeton University, USA

Brown University, USA

North Carolina State University, USA

Tata Inst. of Fund. Research, India

University of Michigan, USA

University of California, Berkeley, USA

Harvard University, USA

Stanford University, USA

Hasselt University, Belgium

Université Paris VII, France

Nihon University, Japan

University of California, San Diego, USA

Princeton University, USA

PRODUCTION

production@msp.org

Silvio Levy, Scientific Editor

See inside back cover or msp.org/ant for submission instructions.

The subscription price for 2013 is US $\$ 200 /$ year for the electronic version, and $\$ 350 /$ year $(+\$ 40$, if shipping outside the US) for print and electronic. Subscriptions, requests for back issues and changes of subscribers address should be sent to MSP.

Algebra \& Number Theory (ISSN 1944-7833 electronic, 1937-0652 printed) at Mathematical Sciences Publishers, 798 Evans Hall \#3840, c/o University of California, Berkeley, CA 94720-3840 is published continuously online. Periodical rate postage paid at Berkeley, CA 94704, and additional mailing offices.

ANT peer review and production are managed by EditFLOW ${ }^{\circledR}$ from Mathematical Sciences Publishers.

\section{PUBLISHED BY}

- mathematical sciences publishers

nonprofit scientific publishing

http://msp.org/

(C) 2013 Mathematical Sciences Publishers 


\section{Algebra \& Number Theory}

Volume $7 \quad$ No. $10 \quad 2013$

On Kato's local $\epsilon$-isomorphism conjecture for rank-one Iwasawa modules

OTMAR VENJAKOB

Polyhedral adjunction theory

SANDRA Di Rocco, Christian HaAse, Benjamin Nill and ANDREas

PAFFENHOLZ

Genericity and contragredience in the local Langlands correspondence

TASHO KALETHA

Homogeneous projective bundles over abelian varieties

MICHEL BRION

On the second Tate-Shafarevich group of a 1-motive

PETER JOSSEN

Triangulable $\mathcal{O}_{F}$-analytic $\left(\varphi_{q}, \Gamma\right)$-modules of rank 2

LIONEL FoURQUAUX and BINGYONG XIE 